\title{
Constraining the Neutron Star Radius with Joint Gravitational-wave and Short Gamma- Ray Burst Observations of Neutron Star-Black Hole Coalescing Binaries
}

\author{
Stefano Ascenzi ${ }^{1,2,3}$ (10), Nicola De Lillo ${ }^{4}$ (i), Carl-Johan Haster ${ }^{5}$ (1) Frank Ohme $^{6,7}$, and Francesco Pannarale ${ }^{8,9,10}$ (1) \\ ${ }^{1}$ Università di Roma Tor Vergata, Via della Ricerca Scientifica 1, I-00133 Roma, Italy \& Sezione INFN di Roma Tor Vergata, I-00133 Roma, Italy \\ 2 INAF-Osservatorio Astronomico di Roma, Monte Porzio Catone (RM), Italy \\ 3 Dip. di Fisica, Università di Roma "Sapienza," P.le A. Moro, 2, I-00185 Rome, Italy \\ ${ }^{4}$ Università di Trento, Dipartimento di Fisica, Via Sommarive 14, I-38123 Povo, Trento, Italy \\ ${ }^{5}$ Canadian Institute for Theoretical Astrophysics, University of Toronto, Toronto, Ontario M5S 3H8, Canada \\ ${ }^{6}$ Max-Planck-Institut für Gravitationsphysik, Albert-Einstein-Institut, Callinstraße 38, D-30167 Hannover, Germany \\ 7 Leibniz Universität Hannover, D-30167 Hannover, Germany \\ ${ }^{8}$ Gravity Exploration Institute, School of Physics and Astronomy, Cardiff University, The Parade, Cardiff CF24 3AA, UK; francesco.pannarale@ligo.org \\ 9 Dipartimento di Fisica, Università di Roma "Sapienza," Piazzale A. Moro 5, I-00185, Rome, Italy \\ ${ }^{10}$ INFN Sezione Roma1, Piazzale A. Moro 5, I-00185, Rome, Italy \\ Received 2018 August 21; revised 2019 March 29; accepted 2019 April 17; published 2019 May 29
}

\begin{abstract}
Coalescing neutron star (NS)-black hole (BH) binaries are promising sources of gravitational-waves (GWs) that are predicted to be detected within the next few years by current GW observatories. If the NS is tidally disrupted outside the $\mathrm{BH}$ innermost stable circular orbit, an accretion torus may form, and this could eventually power a short gamma-ray burst (SGRB). The observation of an SGRB in coincidence with gravitational radiation from an NS-BH coalescence would confirm the association between the two phenomena and also give us new insights into NS physics. We present here a new method to measure NS radii and thus constrain the NS equation of state using joint SGRB and GW observations of NS-BH mergers. We show that in the event of a joint detection with a realistic GW signal-to-noise ratio of 10 , the NS radius can be constrained to $\lesssim 20 \%$ accuracy at $90 \%$ confidence.
\end{abstract}

Key words: binaries: close - equation of state - gamma-ray burst: general - gravitational waves - stars: neutron

\section{Introduction}

The first observation of a binary black hole $(\mathrm{BH})$ merger in GWs made by Advanced LIGO, GW150914, marked the dawn of the GW astronomy era (Abbott et al. 2016b). Subsequently, the LIGO-Virgo Collaboration reported another nine binary $\mathrm{BH}$ merger observations (Abbott et al. 2016a, 2017c, 2017d, 2017 e, 2018b), and the detection of GW170817, a signal that is consistent with a binary NS inspiral (Abbott et al. 2017f). Hinderer et al. (2018) showed that NS-BH systems with certain parameter combinations are also consistent with the GW and electromagnetic (EM) observations of GW170817.

Second-generation GW detectors-i.e., Advanced LIGO (Aasi et al. 2015), Virgo (Acernese et al. 2015), KAGRA (Aso et al. 2013), and LIGO-India (Iyer et al. 2011; Unnikrishnan 2013) — will also be able to detect the GW radiation emitted by NS-BH coalescing binaries, a category of compact binary that remains to be observed. In addition to GWs, among the reasons of interest in coalescing NS-BH binaries is the possibility that if the NS is tidally disrupted outside the innermost stable circular orbit (ISCO) of its $\mathrm{BH}$ companion, matter can be accreted onto the BH, powering a SGRB (Nakar 2007). We now know that a binary NS merger can power an SGRB (Abbott et al. 2017b), and future joint GW-EM observations will be able to determine whether this is true for NS-BH systems too. Naturally, such observations are intrinsically challenging due to the low expected GW-SGRB joint detection rate for NS-BH binaries. This is predicted by Clark et al. (2015) to be $0.4-10 \mathrm{yr}^{-1}$ for LIGO-Virgo at design sensitivity and an idealized SGRB observing facility with all-sky coverage, in line with earlier results from Nissanke et al. (2013) (up to $3 \mathrm{yr}^{-1}$ with a three detector network when ignoring source inclination requirements). The estimate drops to $0.03-0.7 \mathrm{yr}^{-1}$ when considering the Swift field of view. For comparison,
Wanderman \& Piran (2015) calculated joint detection rates with Swift and Fermi of $0.3-1.4 \mathrm{yr}^{-1}$ and $3-10 \mathrm{yr}^{-1}$, respectively, while Regimbau et al. (2015) determined $0.001-0.16 \mathrm{yr}^{-1}$ in the case of Swift. The assumptions behind these frameworks are different and we refer the interested reader to the original articles for details. The upcoming third generation of GW detectors, however, will have a much larger observational horizon (up to $z \simeq 4$ for NS-BH binaries), which automatically increases the joint detection rate considerably (Punturo et al. 2010; Abernathy et al. 2011; Kalogera et al. 2019; Sathyaprakash et al. 2019). Further interest in NS-BH binaries is due to the possibility that the tidally disrupted material is ejected away from the NS-BH system, generating an EM transient powered by the decay of $r$-process ions (macronova) (Li \& Paczyński 1998; Kulkarni 2005; Metzger et al. 2010; Metzger \& Berger 2012; Fernández \& Metzger 2016; Metzger 2017). Similar to the SGRB case, recent GW-EM observations of GW170817 have confirmed that binary NSs are sites that host $r$-processes (Abbott et al. 2017a, $2017 \mathrm{~g}$ ), but whether this holds for NS-BH binaries as well remains to be proven observationally.

Whether the NS in an NS-BH binary undergoes tidal disruption or not, and the amount of matter that is available for accretion (or to feed into the ejecta) in the event of a tidal disruption, both depend on the physical properties of the $\mathrm{BH}$ (mass and spin) and of the NS, including the currently unknown equation of state (EOS) that regulates the microphysics of the NS (Pannarale et al. 2011b; Foucart 2012; Foucart et al. 2018). The GW radiation of coalescing NS-BH systems also depends on the source properties, and among them is the NS EOS (Bildsten \& Cutler 1992; Kokkotas \& Schafer 1995; Vallisneri 2000; Shibata et al. 2009; Duez et al. 2010; Kyutoku et al. 2010, 2011; Lackey et al. 2012, 2014; Foucart et al. 2013, 2014; Pannarale et al. 2013, 2015a, 2015b; 
Kawaguchi et al. 2015; Hinderer et al. 2016; Kumar et al. 2017; Dietrich et al. 2019), but it may be hard to constrain the NS EOS with NS-BH GW inspiral signals only (Pannarale et al. 2011a). Therefore, the GW and EM emission of NS-BH binaries that undergo tidal disruption will carry information about all the properties of the progenitor system, and hence about the NS EOS.

Pannarale \& Ohme (2014) showed how joint GW and SGRB observations of NS-BH coalescences may provide invaluable information about the NS EOS. On the basis of this observation, we propose a method to exploit such observations in order to constrain the NS radius, and thus the NS EOS. In the scenario in which NS-BH systems are progenitors of SGRB central engines, it is reasonable to expect the SGRB energy be proportional to the rest mass of the torus that accretes onto the remnant $\mathrm{BH}$. In turn, this mass can be expressed as a function of the mass and spin of the BH initially present in the binary, and the NS mass and radius (Foucart 2012; Foucart et al. 2018). Our method explores the portion of parameter space that is pinpointed by the $\mathrm{GW}$ observation-GW Bayesian inference provides posterior distributions for the two masses and the $\mathrm{BH}$ spin - and thus determines a posterior distribution for the NS radius by imposing the condition that the merger yields a torus sufficiently massive to power the observed SGRB energy.

Assuming an SGRB isotropic energy of $E_{\gamma, \mathrm{ISO}}=10^{51} \mathrm{erg}$, we expect to be able to measure the NS radius (at $90 \%$ confidence) with $\lesssim 20 \%$ accuracy, given a GW detection with a signal-to-noise ratio $(\mathrm{S} / \mathrm{N})$ of 10. This measure is expected to improve for less energetic SGRBs and GWs with higher S/N. We show that the poorly known parameters that our analysis marginalizes oversuch as the mass-energy conversion efficiency for the SGRBhave a negligible impact on our results, provided the SGRB energy is sufficiently low. Our method is well behaved even for (non-isotropic) energies as high as $E_{\gamma}=10^{50} \mathrm{erg}$, thus the restriction is not very limiting.

The paper is organized as follows. In Section 2 we describe our method in detail, discussing the poorly constrained parameters involved in the analysis. In Section 3 we test the method and present the results we obtained by simulating joint GW-SGRB observations. Finally, in Section 4 we draw our conclusions.

Throughout the paper, we assume geometric units $(G=c=1)$, unless otherwise explicitly noted.

\section{Methodology}

When an NS undergoes tidal disruption during an NS-BH coalescence, part of the matter that constitutes it may remain outside the BH up to a few milliseconds after the merger. We denote the mass of this remnant matter by $M_{\mathrm{rem}}$. A small fraction of this will form unbound ejecta that can eventually power EM transients by radioactive decay of $r$-process heavy ions (Li \& Paczyński 1998; Kulkarni 2005; Metzger et al. 2010; Metzger \& Berger 2012; Fernández \& Metzger 2016; Metzger 2017). The rest of it will stay bound around the BH, forming a neutrino-cooled accretion disk and a tidal tail, orbiting with high eccentricity, which will fall back, filling the disk on a timescale of 0.1-1 s (Foucart 2012). The remnant BH and the disk form a system that is a plausible candidate for the central engine of (a fraction of) SGRBs, as the accretion of mass from the disk onto the BH could power the launch of a relativistic jet (Eichler et al. 1989; Paczynski 1991;
Meszaros \& Rees 1992; Narayan et al. 1992; Mészáros 2006; Lee \& Ramirez-Ruiz 2007).

Given a disk of mass $M_{\text {disk }}$, the energy radiated in gammarays during the prompt emission by conversion of mass corresponds to

$$
E_{\gamma}=\epsilon M_{\mathrm{disk}},
$$

where $\epsilon$ is the mass-energy conversion efficiency. $E_{\gamma}$ is related to the SGRB isotropic energy $E_{\gamma, \text { ISO }}$ by

$$
E_{\gamma}=\left(1-\cos \theta_{\mathrm{j}}\right) E_{\gamma, \mathrm{ISO}},
$$

where $\theta_{\mathrm{j}}$ is the jet half-opening angle, i.e., its beaming angle. ${ }^{11}$ In this work, we assume $E_{\gamma, \text { ISO }}$ to be measured from the gamma-ray flux, provided the distance to the host galaxy of the SGRB is known. We may therefore write

$$
\left(1-\cos \theta_{\mathrm{j}}\right) E_{\gamma, \mathrm{ISO}}=\epsilon M_{\text {disk}} .
$$

Assuming the gravitational radiation emitted by the coalescence is also observed, one can exploit this last equation to connect the measured $E_{\gamma, \mathrm{ISO}}$ and the NS-BH properties inferred from the GW measurement (masses and spins of the binary constituents, as discussed later on in this section) in order to constrain the NS radius, and hence the NS EOS.

Two unknowns are evident in Equation (3). The first one is the efficiency $\epsilon$, which varies from system to system and is determined by a chain of complicated physical processes, the nature of which is an open field of investigation (see, e.g., Nakar 2007 and Lee \& Ramirez-Ruiz 2007, and references therein). The treatment of $\epsilon$ in our analysis is discussed in Section 3.3.1. The second unknown is the beaming angle $\theta_{\mathrm{j}}$. While this can be inferred by measuring the time at which a jet break appears in the afterglow light curve (Sari et al. 1999), usually SGRB jet breaks are not observed and only lower limits $\left(\theta_{\mathrm{j}} \gtrsim 3^{\circ}\right)$ can be placed (Berger 2014). This happens because (i) SGRB afterglows are fainter than long GRB afterglows, and because (ii) their light curves typically drop below a detectable level within a day. We therefore treat $\theta_{\mathrm{j}}$ as an unknown parameter in our analysis, as detailed further in Section 3.3.2.

The last element entering Equation (3) is the disk mass $M_{\text {disk }}$, and we make the approximation $M_{\text {disk }} \simeq M_{\text {rem }}$ (i.e., we neglect the mass of the possible ejecta ${ }^{12}$ ). This approximation is justified by the results of numerical-relativity simulations, which predict ejecta masses of at most $\sim \mathcal{O}\left(10^{-2} M_{\odot}\right)$ (Kawaguchi et al. 2015, 2016; Kyutoku et al. 2015; Foucart et al. 2017) and total remnant masses that are an order of magnitude higher in such extreme cases (Kyutoku et al. 2011; Foucart 2012; Foucart et al. 2017).

We express $M_{\text {rem }}$ using the semi-analytical formula of Foucart et al. (2018), which updates a formula previously introduced in Foucart (2012) and is obtained by fitting results of fully relativistic numerical-relativity simulations. Specifically, the fraction of NS matter that remains outside the

\footnotetext{
11 This expression holds for a simple, top-hat jet model. It can be replaced with a more complicated angle dependency that appropriately models a structured jet.

${ }^{12}$ The observation of the kilonova emission from the same event, or the lack thereof, could be used to constrain the ejecta mass, and therefore to assess the systematics deriving from this approximation.
} 
remnant $\mathrm{BH}$ is given by

$$
\frac{M_{\mathrm{rem}}}{M_{\mathrm{b}, \mathrm{NS}}}=\left[\alpha \frac{1-2 C_{\mathrm{NS}}}{\eta^{1 / 3}}-\beta \hat{R}_{\mathrm{ISCO}} \frac{C_{\mathrm{NS}}}{\eta}+\gamma\right]^{\delta},
$$

where $M_{\mathrm{b}, \mathrm{NS}}$ is the baryonic mass of the NS, $\eta=M_{\mathrm{BH}}$ $M_{\mathrm{NS}} /\left(M_{\mathrm{BH}}+M_{\mathrm{NS}}\right)^{2}$ is the symmetric mass ratio $\left(M_{\mathrm{BH}}\right.$ and $M_{\mathrm{NS}}$ being the gravitational mass of the $\mathrm{BH}$ and the NS, respectively), $R_{\mathrm{NS}}$ is the radius of the NS at isolation expressed in Schwarzschild coordinates, $C_{\mathrm{NS}}=M_{\mathrm{NS}} / R_{\mathrm{NS}}$ is the NS compactness, $\chi_{\mathrm{BH}}$ is the dimensionless spin magnitude of the $\mathrm{BH}$ in the NS-BH binary, $\hat{R}_{\mathrm{ISCO}}=R_{\mathrm{ISCO}} / M_{\mathrm{BH}}$ is the normalized ISCO radius, and $\alpha=0.406, \beta=0.139, \gamma=0.255$, $\delta=1.761$ are the free coefficients determined by the fitting procedure. ${ }^{13}$ The ISCO radius $R_{\mathrm{ISCO}}$ is a function of the mass $M_{\mathrm{BH}}$ and spin magnitude $\chi_{\mathrm{BH}}$ of the $\mathrm{BH}$ in the original NS-BH binary (Bardeen et al. 1972).

The discussion carried out so far can be summarized as follows: an NS-BH coalescence can result in an SGRB with energy proportional to the rest mass liberated by the tidal disruption and given by Equation (4). The system of equations laid out is closed by prescribing an EOS for the NS. This enters the expression(s) for the remnant mass through $R_{\mathrm{NS}}$ and $M_{\mathrm{b}, \mathrm{NS}}$. Given that our goal is to determine a method to constrain the NS EOS on the basis of a joint GW-SGRB observation of an NS-BH coalescence, the EOS is ultimately the unknown we would want to solve for, under the constraints imposed by the observational data. In order to simplify this task and to avoid repeatedly solving the TolmanOpenheimer-Volkoff NS structure equations (Oppenheimer \& Volkoff 1939; Tolman 1939), we express the NS baryonic mass $M_{\mathrm{b}, \mathrm{NS}}$ as a function of the NS gravitational mass $M_{\mathrm{NS}}$ and solve for $R_{\mathrm{NS}}$. In this sense, our method constrains the NS radius and indirectly constrains the NS EOS.

The approximation we use to relate $M_{\mathrm{NS}}$ to $M_{\mathrm{b}, \mathrm{NS}}$ is the fit to NS equilibrium sequences provided by Cipolletta et al. (2015):

$$
\frac{M_{\mathrm{b}, \mathrm{NS}}}{M_{\odot}}=\frac{M_{\mathrm{NS}}}{M_{\odot}}+c_{2}\left(\frac{M_{\mathrm{NS}}}{M_{\odot}}\right)^{2} .
$$

The value of the free coefficient $c_{2}=13 / 200$ found by Cipolletta and collaborators is biased by their choice of EOSs used to build the NS equilibrium sequences they fit with Equation (5). We find that, for a large sample of EOSs, acceptable values of $c_{2}$ lie in the range [12/200, 23/200] as shown in Figure 1, where we only show six representative EOSs to avoid overcrowding the figure.

Given the observation of an NS-BH coalescence, GW parameter estimation provides posterior probability distributions for the gravitational masses and the $\mathrm{BH}$ spin that enter Equation (4). Once we obtain the raw posterior distribution samples from the GW analysis, we "prune" them as follows. We discard all parameter points that do not satisfy the requirements $M_{1}>3 M_{\odot}$ (i.e., the primary object is presumably not a $\mathrm{BH}$ because it is not massive enough), $M_{2}<2.8 M_{\odot}$, and $\chi_{2}<0.4$ (i.e., the secondary object is presumably not an NS because its mass and/or spin are too high). ${ }^{14}$ This step

\footnotetext{
${ }^{13}$ We omit the max between 0 and the term in square brackets of Equation (4) that appears in the original expression for $\frac{M_{\mathrm{rem}}}{M_{\mathrm{b}, \mathrm{NS}}}$ given in Foucart et al. (2018). The reason for this is explained in Section 3.

${ }^{14}$ This is the only step where the information on the spin of the secondary object is exploited.
}

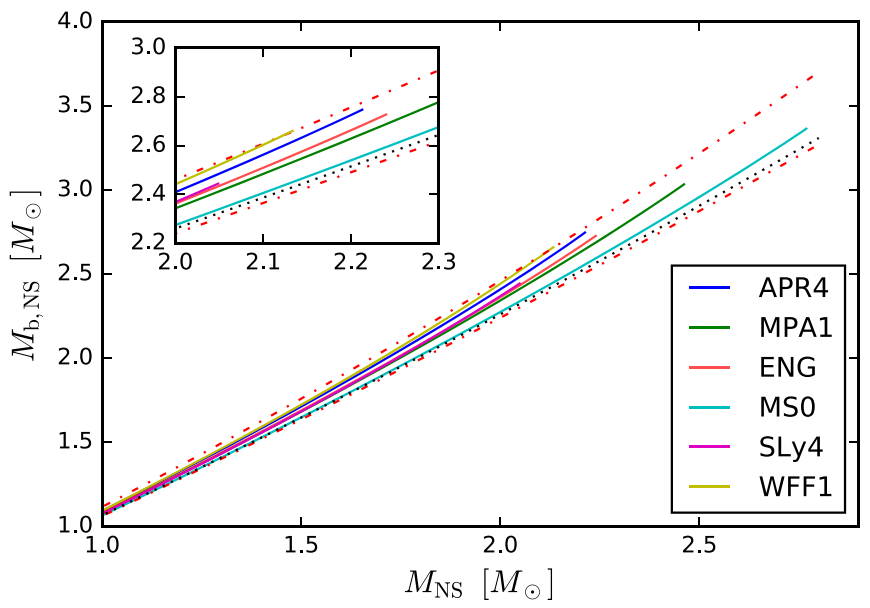

Figure 1. Baryonic-gravitational mass relations along stable NS equilibrium sequences obtained with different EOSs (continuous curves). The black dotted line is the fit in Equation (5) with its original value $c_{2}=13 / 200$ Cipolletta et al. (2015). The red dotted-dashed lines correspond instead to $c_{2}=\{12 / 200$, $23 / 200\}$ (lower and upper curve, respectively). These two values allow us to enclose all the NS equilibrium sequences.

allows us to downsample the posteriors of the GW measurement to a set of points reasonably compatible with the assumption that the observed SGRB was due to an NS-BH progenitor.

After the pruning of the GW posteriors, we determine a posterior for the NS radius $R_{\mathrm{NS}}$ as follows. We randomly sample the joint $\mathrm{GW}$ posterior distribution for $M_{\mathrm{NS}}, M_{\mathrm{BH}}$ and $\chi_{\mathrm{BH}}$ (effectively using it as an informed prior in a hierarchical analysis), and assume uniform prior distributions for $R_{\mathrm{NS}}$ and the remaining unknowns in our setup, i.e., $\epsilon, c_{2}$, and $\cos \theta_{\mathrm{j}}$. From Equation (3) we thus obtain a distribution for $E_{\gamma, \text { ISO }}$. Each value of this distribution is then compared to $E_{\gamma, \text { ISO }}^{\text {obs }}$ which is the measured value of $E_{\gamma, \text { ISO }}$. We then reject any sample point that yields an energy that differs by more than a given tolerance $\tau$ from the observed $E_{\gamma, \text { ISO }}$, according to the condition

$$
\frac{\left|E_{\gamma, \text { ISO }}-E_{\gamma, \text { ISO }}^{\text {obs }}\right|}{E_{\gamma, \text { ISO }}^{\text {obs }}}>\tau .
$$

Here $\tau$ accounts both for an uncertainty on the observed SGRB energy and for errors introduced using the approximate formula in Equation (4), which Foucart et al. (2018) reported to be $\sim 15 \%$. We also reject any sample point that yields a violation of the causality condition $R_{\mathrm{NS}} \geqslant 3.04 G M_{\mathrm{NS}} / c^{2}$ (Lattimer et al. 1990; Glendenning 1992).

It is important to stress that although the GW signal alone can bring information on $R_{\mathrm{NS}}$ (e.g., via the tidal deformability of the star), this information is not exploited by our method, in order keep our analysis as agnostic as possible. While this constitutes a loss of information, we avoid introducing systematic errors due to the modeling of the EOS imprints on the $\mathrm{GW}$ signal waveform. We will explore the benefits of using the full GW information in a future study.

The building blocks of our method are summarized as follows:

1. Parameter estimation on the GW signal to obtain posterior distribution on the signal parameters. Among these, $M_{1}$ (mass of the primary star), $M_{2}$ (mass of the 
Table 1

Parameters Describing the Joint GW-SGRB Observation Scenarios Considered in This Work

\begin{tabular}{lcccccc}
\hline \hline Label & $\begin{array}{c}M_{\mathrm{NS}} \\
\left(M_{\odot}\right)\end{array}$ & $\begin{array}{c}M_{\mathrm{BH}} \\
\left(M_{\odot}\right)\end{array}$ & $\chi_{\mathrm{BH}}$ & $\begin{array}{c}E_{\gamma, \mathrm{ISO}}^{\mathrm{obs}} \\
\left(10^{50} \mathrm{erg}\right)\end{array}$ & $\begin{array}{c}R_{\mathrm{NS}} \\
(\mathrm{km})\end{array}$ & $\begin{array}{c}\mathcal{M}_{\mathrm{c}} \\
\left(M_{\odot}\right)\end{array}$ \\
\hline m484chi048L & 1.35 & 4.84 & 0.48 & 1 & 10.124 & 2.14 \\
m484 chi048H & 1.35 & 4.84 & 0.48 & 50 & 10.521 & 2.14 \\
m484 chi080L & 1.35 & 4.84 & 0.80 & 1 & 7.797 & 2.14 \\
m484 chi080H & 1.35 & 4.84 & 0.80 & 50 & 8.103 & 2.14 \\
m100chi070L & 1.35 & 10.0 & 0.70 & 1 & 11.183 & 2.93 \\
m100chi070H & 1.35 & 10.0 & 0.70 & 50 & 11.569 & 2.93 \\
\hline
\end{tabular}

Note. Each case is labeled by the $\mathrm{BH}$ mass and spin, while the last letter refers to the SGRB (simulated) observed isotropic energy $(\mathrm{L} / \mathrm{H}$ for low $/$ high). The NS radius $R_{\mathrm{NS}}$ is determined from Equation (7) after setting $c_{2}=17 / 200$, $\cos \theta_{\mathrm{j}}=0.98$, and $\epsilon=0.01$. All masses are defined in their respective source frame.

secondary star), and $\chi_{1}$ (spin of the primary star) are those that will directly enter in our analysis. An example of this is provided in Section 3.2.

2. The posterior distribution of $\mathrm{GW}$ signal parameters is "pruned" to reject all the parameter combinations incompatible with a NS-BH system. The criterion involves conditions on $M_{1}, M_{2}$, and $\chi_{2}$ (spin of the secondary star) described earlier in this section. After this step we can set $M_{1}=M_{\mathrm{BH}}, M_{2}=M_{\mathrm{NS}}$, and $\chi_{1}=\chi_{\mathrm{BH}}$.

3. We use the distributions of $M_{\mathrm{NS}}, M_{\mathrm{BH}}$ and $\chi_{\mathrm{BH}}$ as $\mathrm{GW}$ informed priors. We define priors for the other parameters $\theta_{\mathrm{j}}, \epsilon, c_{2}$, and $R_{\mathrm{NS}}$ (an example of this is reported in Section 3.3). Sampling $N$ times over the joint distribution of $M_{\mathrm{NS}}, M_{\mathrm{BH}}, \chi_{\mathrm{BH}}, \theta_{\mathrm{j}}, \epsilon, c_{2}$, and $R_{\mathrm{NS}}$, we can solve Equation (3) (with $M_{\text {rem }}$ provided by Equation (4)) for each sample point in order to obtain a posterior distribution on $E_{\gamma, \text { ISO }}$.

4. We reject all sample points that do not satisfy the condition in Equation (6) as well as the causality constraint in order to obtain a posterior distribution on $R_{\mathrm{NS}}$.

The next section presents the application of our method to several simulated joint GW-SGRB observations.

\section{Method Performance Assessment}

\subsection{Injection of the Signal}

To assess the performance of our method, we simulate various joint GW-SGRB observations of NS-BH coalescing binaries characterized by the sets of parameters reported in Table 1. The "true" reference value of the NS radius-i.e., the quantity that our method aims at recovering - is determined by solving Equation (4) for $R_{\mathrm{NS}}$, once the parameters $M_{\mathrm{NS}}, M_{\mathrm{BH}}$, $\chi_{\mathrm{BH}}$, and $E_{\gamma, \mathrm{ISO}}$ of the simulated observation are specified:

$$
R_{\mathrm{NS}}=\frac{\left(2 \alpha \eta^{-1 / 3}+\beta \hat{R}_{\mathrm{ISCO}} \eta^{-1}\right) M_{\mathrm{NS}}}{\alpha \eta^{-1 / 3}+\gamma-\left[E_{\gamma} /\left(\epsilon M_{\mathrm{b}, \mathrm{NS}}\right)\right]^{1 / \delta}} .
$$

Here we also substituted $E_{\gamma} / \epsilon$ for $M_{\text {rem }}$.

The three remaining free parameters are set to $c_{2}=17 / 200$, $\cos \theta_{\mathrm{j}}=0.98$, i.e., $\theta_{\mathrm{j}} \simeq 11^{\circ}$, and $\epsilon=0.01$, which are all within their respective prior distribution ranges. These choices do not affect the final outcome of our analysis, but only serve the purpose of providing a target value for the NS radius.
The properties of the simulated NS-BH coalescences are given in Table 1 with masses specified in their respective source frame, the $\mathrm{BH}$ spin $\chi_{\mathrm{BH}}$ being aligned with the orbital angular momentum and assuming the NS is non-spinning. We also assume alignment between the total angular momentum and the line of sight, consistent with an observation of both GWs and an SGRB jet. To highlight the capabilities of the analysis presented in this paper, and to remove sources of both systematic and statistical uncertainties, the GW signal is injected into a data stream without added Gaussian noise, and both the injected signal and the parameter estimation analysis are using the IMRPHENOMPV2 GW model (Hannam et al. 2014; Husa et al. 2016; Khan et al. 2016; Smith et al. 2016). This model includes an effective treatment of the spin-precession dynamics, but does not take the imprint of possible NS tidal disruptions onto the $\mathrm{GW}$ signal into account. Thus, the $R_{\mathrm{NS}}$ constraints presented in this study can be taken as lower bounds, as further direct information about the NS properties should only act to narrow these constraints.

As reported in Table 1, for each of the three NS-BH systems we consider, we use two values of the isotropic energy. This allows us to assess how this quantity affects the measurement of $R_{\mathrm{NS}}$. We inject the NS-BH GW signals at two values of $\mathrm{S} / \mathrm{N}$, namely 30 and 10 , which correspond to sources at redshift $z \simeq 0.04$ and $z \simeq 0.12$, respectively.

\subsection{Recovery of the GW Signal and Parameter Estimation}

The parameter estimation of the GW signal is performed using the LALINFERENCE package (Veitch et al. 2015; LIGO Scientific Collaboration, Virgo Collaboration 2017) assuming a detector network consisting of LIGO-Hanford and LIGOLivingston, both operating at their nominal design sensitivities (Abbott et al. 2013; Aasi et al. 2015).

In the parameter estimation analysis we perform an "agnostic" recovery, where we assume a prior distribution on the detector-frame masses as uniform within [1.0, 14.3] $M_{\odot}$, with additional constraints on both the (gravitational) mass ratio $\left[1 \leqslant M_{\mathrm{NS}} / M_{\mathrm{BH}} \leqslant 1 / 8\right]$ and chirp mass, $\mathcal{M}_{c}=$ $\left(M_{\mathrm{BH}} M_{\mathrm{NS}}\right)^{3 / 5}\left(M_{\mathrm{BH}}+M_{\mathrm{NS}}\right)^{-1 / 5}$, within $[2.18,4.02] M_{\odot} . \mathrm{We}$ allow for isotropically distributed spins with dimensionless spin magnitudes of $[0 \leqslant \chi \leqslant 0.89]$ for both binary objects, but as the injected binary is viewed face-on we expect only a minimal information contribution from the binaries' spinprecession (Fairhurst 2018). The analysis assumes a uniformin-volume distribution for the sources' luminosity distance, and because we require a joint GW-SGRB observation we assume the direction of the SGRB as known and fix the sky location to its true values in the GW analysis. Finally, we allow for isotropically oriented binaries, with no restrictions on the binary inclination or constraints from the allowed beaming angles in the GW analysis itself. The results of the parameter estimation on the GW injected signals are summarized in Table 2 in the Appendix, where the $90 \%$ of credible intervals on the masses, the spin of the primary star, and the mass ratio $q$ are reported. In Table 3 we also reported on the $90 \%$ intervals for the same quantities obtained after the pruning of the posteriors.

\subsection{Prior Distributions for the Remaining Parameters}

As discussed in Section 2, values of the parameters $\epsilon, \theta_{\mathrm{j}}, c_{2}$, and $R_{\mathrm{NS}}$ must be provided in order to solve Equation (3) to 
obtain $E_{\gamma, \text { ISO }}$. These are sampled from the prior distributions defined in this dedicated section.

\subsubsection{Prior Distribution for $\epsilon$}

The efficiency $\epsilon$ introduced in Equation (1) is poorly constrained. It can be expressed as the product of $\epsilon_{\text {jet }}$, which is the efficiency of conversion of accreted rest mass into jet kinetic energy, and $\epsilon_{\gamma}$, which is the conversion efficiency from jet kinetic energy to gamma-ray radiation. Zhang et al. (2007) measured the latter efficiency for a sample of long and short Swift GRBs finding values between $30 \%$ and $60 \%$, with an average of $49 \%$. The efficiency $\epsilon_{\text {jet }}$ is not directly measurable and depends on the nature of the jet launching mechanism. This can be driven by magnetohydrodynamics (Blandford \& Znajek 1977; Blandford \& Payne 1982; Parfrey et al. 2015) or by neutrino-antineutrino pair annihilation (Eichler et al. 1989; Zalamea \& Beloborodov 2011). In both cases its value depends upon the spin of the remnant BH (Zalamea \& Beloborodov 2011; Parfrey et al. 2015). In a context similar to ours, Giacomazzo et al. (2013) used a value of $\epsilon=$ $\epsilon_{\gamma} \times \epsilon_{\text {jet }}=0.05$. In our analysis, we draw random values of $\epsilon$ according to a uniform prior distribution between 0 and 0.2 (according to Lee \& Ramirez-Ruiz 2007 it is unlikely for mass to be converted into energy with an efficiency higher than $\sim 0.1$ ).

It is worth noting that, at a given energy $E_{\gamma}$, there is a degeneracy between the NS radius and $\epsilon$. Physically, one can think of the system being able to increase/decrease $E_{\gamma}$ by increasing/decreasing its $\epsilon$ or $M_{\text {disk }}$. The latter may in turn be obtained with an increase/decrease in $R_{\mathrm{NS}}$. To understand how a specific $\epsilon$ may affect the inferred value of $R_{\mathrm{NS}}$, we refer to Equation (7), where we can see that $R_{\mathrm{NS}}$ is roughly independent of $\epsilon$ for $\epsilon \gg E_{\gamma} / M_{\mathrm{b}, \mathrm{NS}}$. If we consider an NS with $M_{\mathrm{b}, \mathrm{NS}} \sim$ $1.5 M_{\odot}$, powering SGRBs with energies $E_{\gamma}=\left\{10^{49}, 10^{50}\right.$, $\left.10^{51}\right\}$ erg would require efficiencies $\epsilon \gg\left\{10^{-4}, 10^{-3}, 10^{-2}\right\}$ in order for the inferred value of $R_{\mathrm{NS}}$ to not be significantly affected. These efficiency values are at most of the same order of magnitude as the ones inferred for the magnetohydrodynamics mechanisms considered in Hawley \& Krolik (2006) and Parfrey et al. (2015), which inspired Giacomazzo et al. (2013) to adopt the fiducial value of $\epsilon=5 \%$. The efficiency for the neutrino-antineutrino annihilation mechanism is expected to be lower, in general, but values of the same order as for the magnetohydrodynamics mechanisms have been found for high BH spins and mass accretion rates (Setiawan et al. 2004; Zalamea \& Beloborodov 2011). Nevertheless, in order to power an SGRB with a remnant mass value up to $\mathcal{O}\left(0.1 M_{\odot}\right)$ (Kyutoku et al. 2011; Foucart 2012; Foucart et al. 2017), the efficiency cannot be lower than $10^{-6}$. Thus, the dependency of $R_{\mathrm{NS}}$ on $\epsilon$ is expected to be weak for faint events even in the case of neutrino-antineutrino pair annihilation.

Finally, if $E_{\gamma, \text { ISO }} \lesssim 10^{50} \mathrm{erg}$, the dependency of $R_{\mathrm{NS}}$ on the beaming angle and $c_{2}$ is also weak, because the term

$$
\frac{E_{\gamma}}{\epsilon M_{\mathrm{b}, \mathrm{NS}}}=\frac{\left(1-\cos \theta_{\mathrm{j}}\right) E_{\gamma, \mathrm{ISO}}}{\epsilon\left(M_{\mathrm{NS}}+c_{2} M_{\mathrm{NS}}^{2} / M_{\odot}\right)}
$$

in the denominator of Equation (7) becomes negligible. Therefore, in this circumstance, our results will not depend on the particular prior distribution choices for $c_{2}$ and $\theta_{\mathrm{j}}$.

\subsubsection{Prior Distribution for $\theta_{j}$}

The information about SGRB beaming angles is sparser than that for long GRBs. The Berger (2014) review, for example, reported a mean beaming angle of $\left\langle\theta_{\mathrm{j}}\right\rangle \gtrsim 10^{\circ}$ for SGRBs and clearly shows how this angle is measured only in a handful of cases. The maximum measured value of $\theta_{\mathrm{j}}$ is about $25^{\circ}$, which was obtained in a single instance. In this work, we therefore consider a cosine-flat prior distribution for $\theta_{\mathrm{j}}$, with angle values limited to the range $\left[1^{\circ}, 30^{\circ}\right]$. However, we note that additional EM follow-up observations of a specific NS-BH coalescence event and its host galaxy could potentially further constrain the sampling interval for $\theta_{\mathrm{j}}$. Finally, it is worth noticing that, concerning the GW side, it is unlikely to measure the inclination of the binary system with a precision that allows us to constrain $\theta_{\mathrm{j}}$ (assuming it is less than $\sim 50^{\circ}$ ) (Fairhurst 2018).

\subsubsection{Prior Distributions for $\mathrm{R}_{N S}$ and $\mathrm{c}_{2}$}

While the NS EOS binds together the values of $R_{\mathrm{NS}}$ and $c_{2}$ at a fundamental level, we use a simplified setup in which both (unknown) quantities are sampled from two independent uniform prior distributions. Our uniform prior distribution for the NS radius runs from 9 to $15 \mathrm{~km}$. This range encompasses the known limits on NS radii that come from observational and theoretical constraints (for reviews on this topic, see Özel \& Freire 2016 and Lattimer \& Prakash 2016), as well as the limits inferred from the analysis of the tidal effects of GW170817 (Abbott et al. 2018a). As stated previously, we found that Equation (5) can accommodate a large set of NS equilibrium sequences built upon different EOSs, provided that $c_{2}$ is allowed to vary between $12 / 200$ and $23 / 200$. In order to be as agnostic as possible about the EOS of NS matter, we adopt a uniform distribution for the unknown $c_{2}$ over such an interval. The impact of this prior on our results is negligible, which lends support the our simplification of sampling $c_{2}$ and $R_{\mathrm{NS}}$ independently. This is due to the fact that $c_{2}$ enters Equation (7) via the NS baryonic mass $M_{\mathrm{b}, \mathrm{NS}}$ (see Equation (5)) in a term that is of the form $\epsilon c_{2} M_{\mathrm{NS}}{ }^{2}$. This term is clearly dominated by the prior on $\epsilon$, which is a truly unknown parameter, and $M_{\mathrm{NS}}$, which is constrained by the $\mathrm{GW}$ analysis.

\subsection{Results}

Given the results of the GW parameter estimation analysis and the pruning of these results to account only for NSBH systems, we sample $N$ points ${ }^{15}$ of the mass and spin pruned posterior distributions to obtain parameters that we feed into Equation (3), which we then solve for $E_{\gamma, \text { ISO }}$ (under the $M_{\text {disk }} \simeq M_{\text {rem }}$ approximation in Section 2). Equation (4) can be used to determine $M_{\mathrm{rem}}$ as a function of the NS-BH parameters.

Once this step is complete, each of the $N$ sample points of the (pruned) $\mathrm{GW}$ posterior is associated with a value of $E_{\gamma, \text { ISO }}$. We can then use the condition given in Equation (6) with $\tau \equiv 2$ to determine the subset of sample points with combinations of parameters such that the energy $E_{\gamma, \text { ISO }}$ they return lies within a $200 \%$ relative difference from the observed energy $E_{\gamma, \text { ISO }}^{\text {obs }}$ The absolute value that appears in Equation (6) allows for combinations of the parameters $M_{\mathrm{BH}}, M_{\mathrm{NS}}$, and $\chi_{\mathrm{BH}}$ that yield

\footnotetext{
15 Typically, we set $N=3 \times 10^{6}$ for cases with $E_{\gamma, \text { ISO }}=10^{50} \mathrm{erg}$ and $N=$ $10^{5}$ for cases with $E_{\gamma, \text { ISO }}=5 \times 10^{51} \mathrm{erg}$.
} 

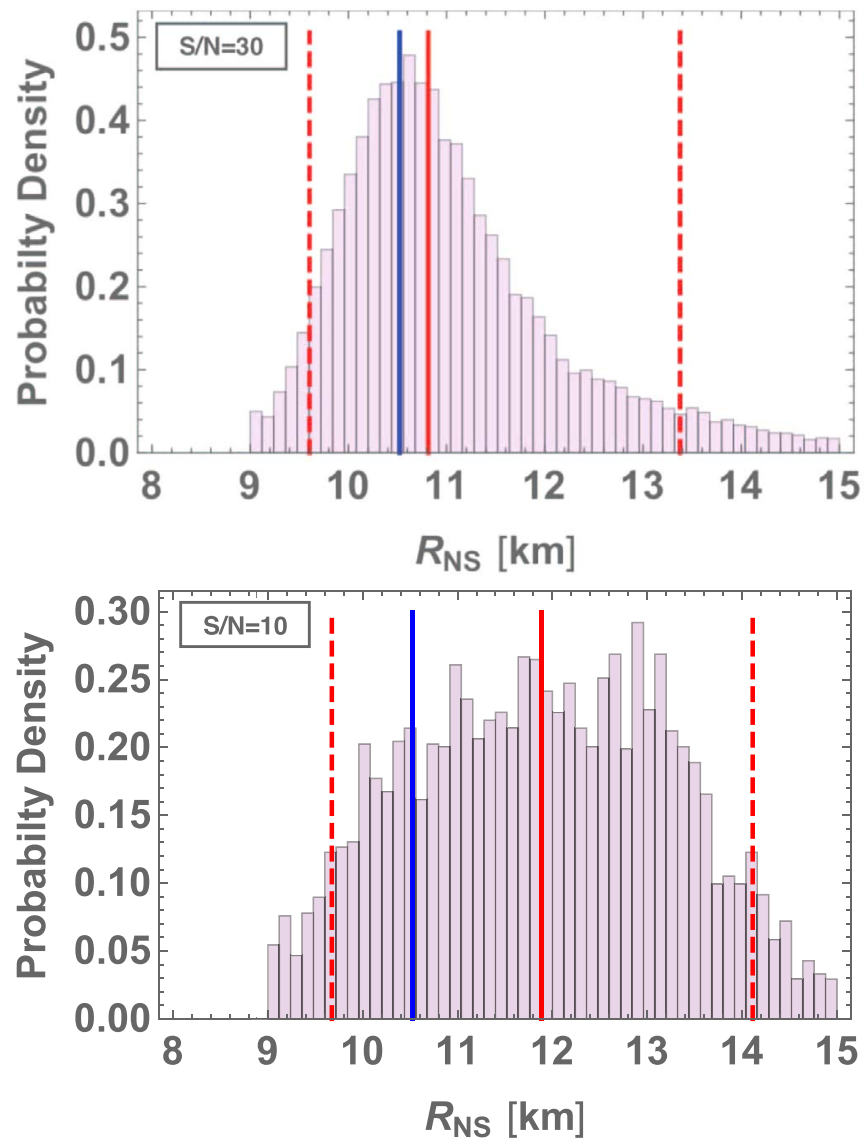

Figure 2. $R_{\mathrm{NS}}$ posterior distribution for case $\mathrm{m} 484 \mathrm{chi} 048 \mathrm{H}$ at $\mathrm{S} / \mathrm{N}=30$ (top panel) and $\mathrm{S} / \mathrm{N}=10$ (bottom panel). The red line indicates the median value of the posterior, while the red dashed lines mark the $90 \%$ credible interval. The blue line represents the injected value of the radius.

a non-physical remnant mass and hence a non-physical $E_{\gamma, \text { ISO }}$. Accepting non-physical remnant masses-rather than setting the hard cut $M_{\text {rem }}=0$ present in the original formulation of Foucart et al. (2018) whenever Equation (4) yields a nonphysical value-corresponds to introducing an uncertainty on the $M_{\text {rem }}=0$ boundary pinpointed by the fitting formula for $M_{\text {rem. }}$.

Figure 2 shows the $R_{\mathrm{NS}}$ posterior distribution obtained for case $\mathrm{m} 484 \mathrm{chi} 048 \mathrm{H}$ (i.e., $M_{\mathrm{BH}}=4.84 M_{\odot}, M_{\mathrm{NS}}=1.35 M_{\odot}$, $\left.\chi_{\mathrm{BH}}=0.48, E_{\gamma, \mathrm{ISO}}=5 \times 10^{51} \mathrm{erg}\right)$ : the top and bottom panel correspond to $\mathrm{S} / \mathrm{N}=30$ and $\mathrm{S} / \mathrm{N}=10$, respectively. The blue solid line marks the target value of the radius, while the red solid line marks $\lambda$, the median of the posterior. Finally, the red dashed lines mark the 5th and 95th percentiles of the posterior distribution $\left(\lambda_{-}, \lambda_{+}\right.$, with $\left.\lambda_{-}<\lambda_{+}\right)$, which enclose the $90 \%$ credible interval. With this choice, the statistical error on the measurement is given by

$$
\sigma_{\text {Stat }} \equiv \frac{\lambda_{+}-\lambda_{-}}{2 \lambda}
$$

We see that the $90 \%$ credible interval encloses the target value of $R_{\mathrm{NS}}$ and that, as expected, it decreases as the $\mathrm{S} / \mathrm{N}$ increases. Similarly, the difference between the injected value of $R_{\mathrm{NS}}$ and the median of the $R_{\mathrm{NS}}$ posterior decreases with increasing $\mathrm{S} / \mathrm{N}$. These dependencies on $\mathrm{S} / \mathrm{N}$ are a sign of the impact that our $\mathrm{GW}$-informed prior for $M_{\mathrm{NS}}, M_{\mathrm{BH}}$, and $\chi_{\mathrm{BH}}$ has on the final
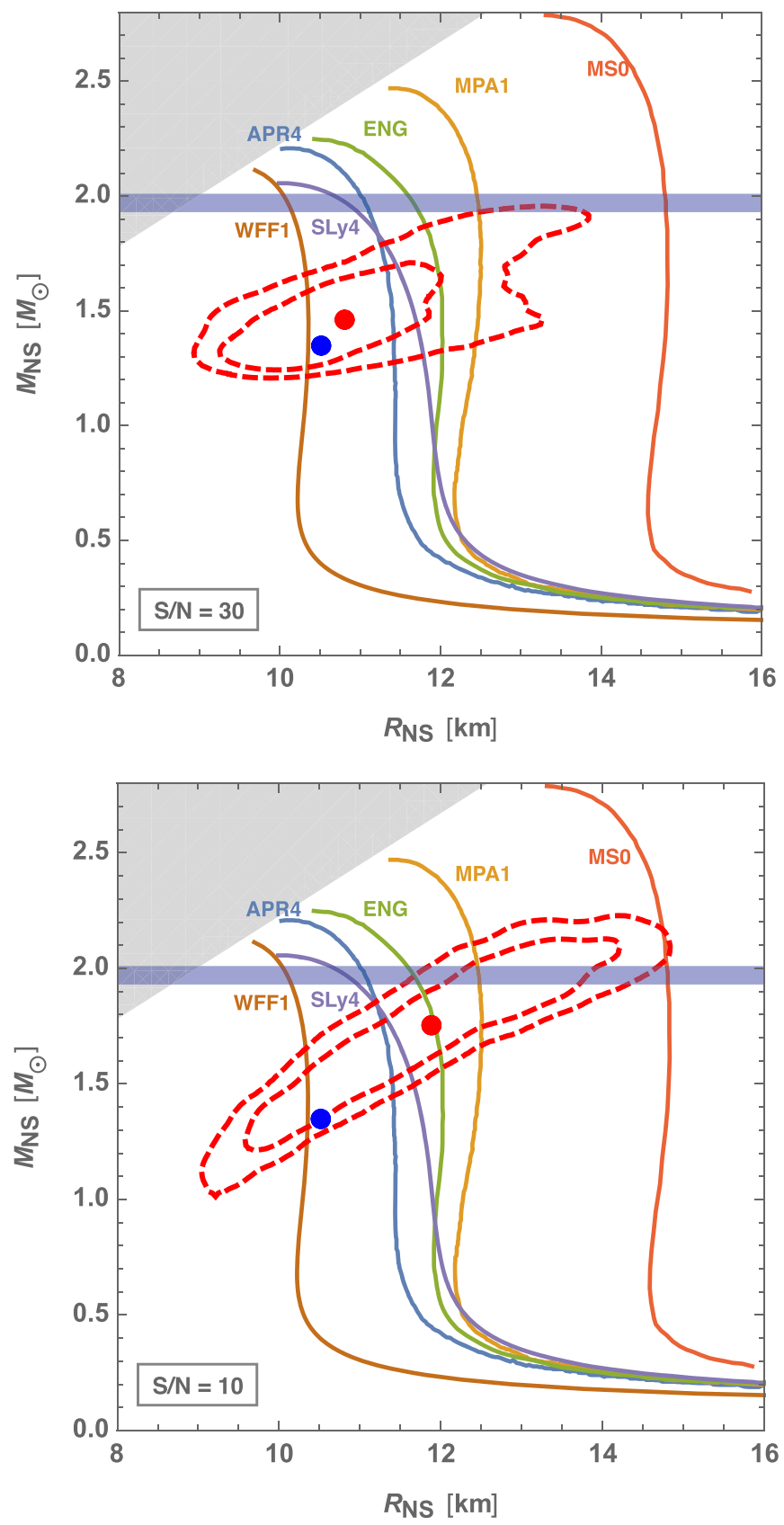

Figure 3. NS mass and radius constraints obtained with our method for case $\mathrm{m} 484 \mathrm{chi} 048 \mathrm{H}$ and $\mathrm{S} / \mathrm{N}=30$ and $\mathrm{S} / \mathrm{N}=10$ in the top and bottom panel, respectively. NS equilibrium sequences for different NS EOSs are also shown. The shaded gray region discards mass-radius combinations excluded by the causality constrain. The horizontal blue band denotes the mass of the high-mass NS J1614-2230 (Demorest et al. 2010). The dashed red lines represent the $68 \%$ and $90 \%$ credible regions. The blue dot marks the injected mass and radius values, while the red dot denotes the values recovered by the analysis as the median of the mass and radius distributions.

results of our approach. We will return to this point in Section 3.5.

In Figure 3, the results for case $\mathrm{m} 484 \mathrm{chi048 \textrm {H }}$ are displayed in the $M_{\mathrm{NS}}-R_{\mathrm{NS}}$ plane and overlaid on NS equilibrium sequences obtained with the APR4 (Akmal et al. 1998), ENG (Engvik et al. 1996), MPA1 (Müther et al. 1987), MS0 (Müller \& Serot 1996), SLy4 (Chabanat et al. 1998), and WFF1 (Wiringa et al. 1988) NS EOSs. Here the gray shaded area denotes the region of the $M_{\mathrm{NS}}-R_{\mathrm{NS}}$ plane where the 

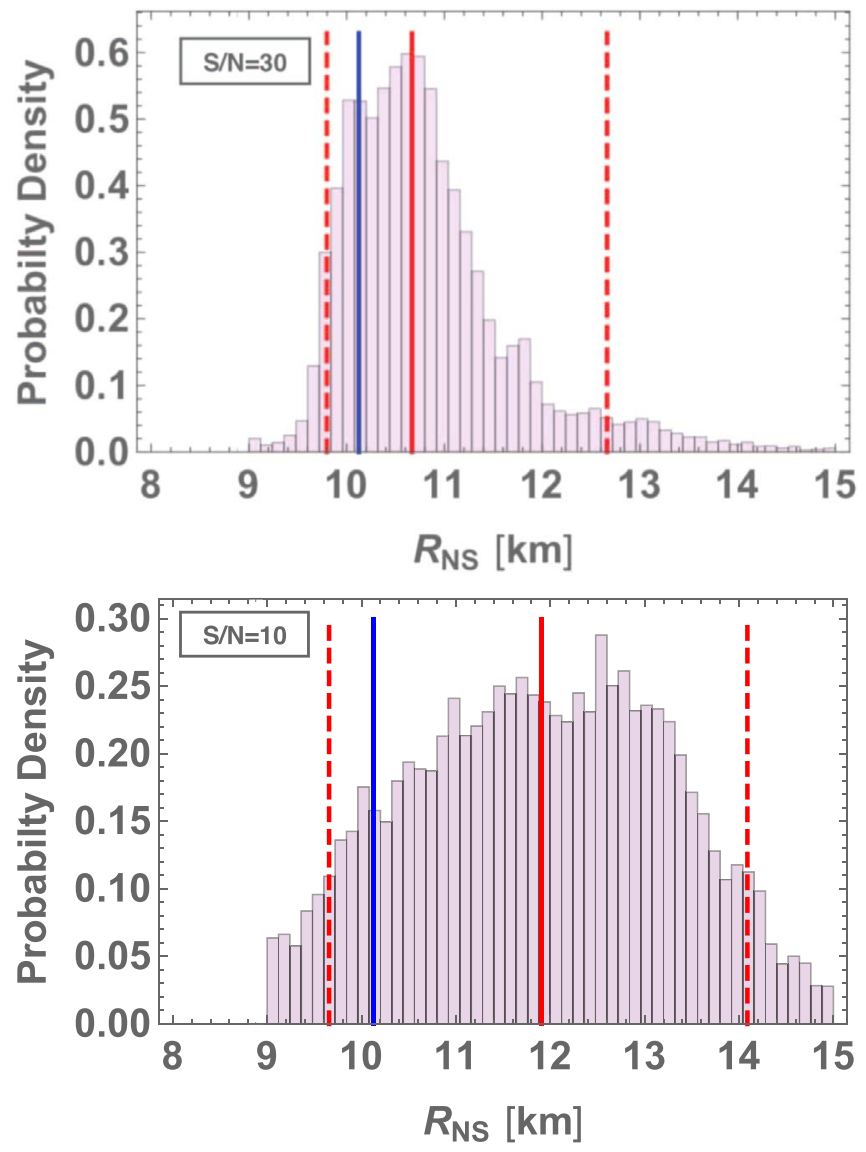

Figure 4. Same as Figure 2 but for case m484chi 048L.

causality constrain is not satisfied, while the blue horizontal band reports the mass of the millisecond pulsar J1614-2230, one of the NSs with the highest mass observed (Demorest et al. 2010). We can see that all the EOSs considered in this figure can account for this high value of mass. The red dashed contours represent the $68 \%$ and $90 \%$ credible regions. As expected, this region shrinks as the $\mathrm{S} / \mathrm{N}$ increases, while still including the injected values of mass and radius (blue dot).

Similar results hold for case $\mathrm{m} 484 \mathrm{chi} 048 \mathrm{~L}$ and are shown in Figures 4 and 5. The decrease in SGRB energy causes the high-end tails of the $R_{\mathrm{NS}}$ distribution to be slightly less populated with respect to the $\mathrm{m} 484 \mathrm{chi} 048 \mathrm{H}$ case. This is not surprising: powering a more energetic SGRB requires a more massive torus, and lower values of $\epsilon$ can accommodate larger values of $R_{\mathrm{NS}}$ in such a scenario. In turn, this means that the impact of the prior on $\epsilon$ progressively increases with the SGRB energy.

This can be further understood from Figure 6, where the recovered posterior distributions for the high-energy case m484chi048H (top panel) and the low-energy case m484chi048L (bottom panel) are compared in the $\epsilon-R_{\mathrm{NS}}$ plane $^{16}$ at $\mathrm{S} / \mathrm{N}=30$. The red dot marks the simulated scenario, while the white, dashed lines denote the $68 \%$ and $90 \%$ credible regions. In the low-energy case, the distribution is populated in regions with $\epsilon \lesssim 10^{-3}$, so the overall weight of high $R_{\mathrm{NS}}$ values is reduced with respect to the high-energy

\footnotetext{
${ }^{16}$ We focus on this specific marginalization of the full results, because $\epsilon$ is the most influential among the unknown parameters that enter our method, and at the same time the least constrained by observations.
}
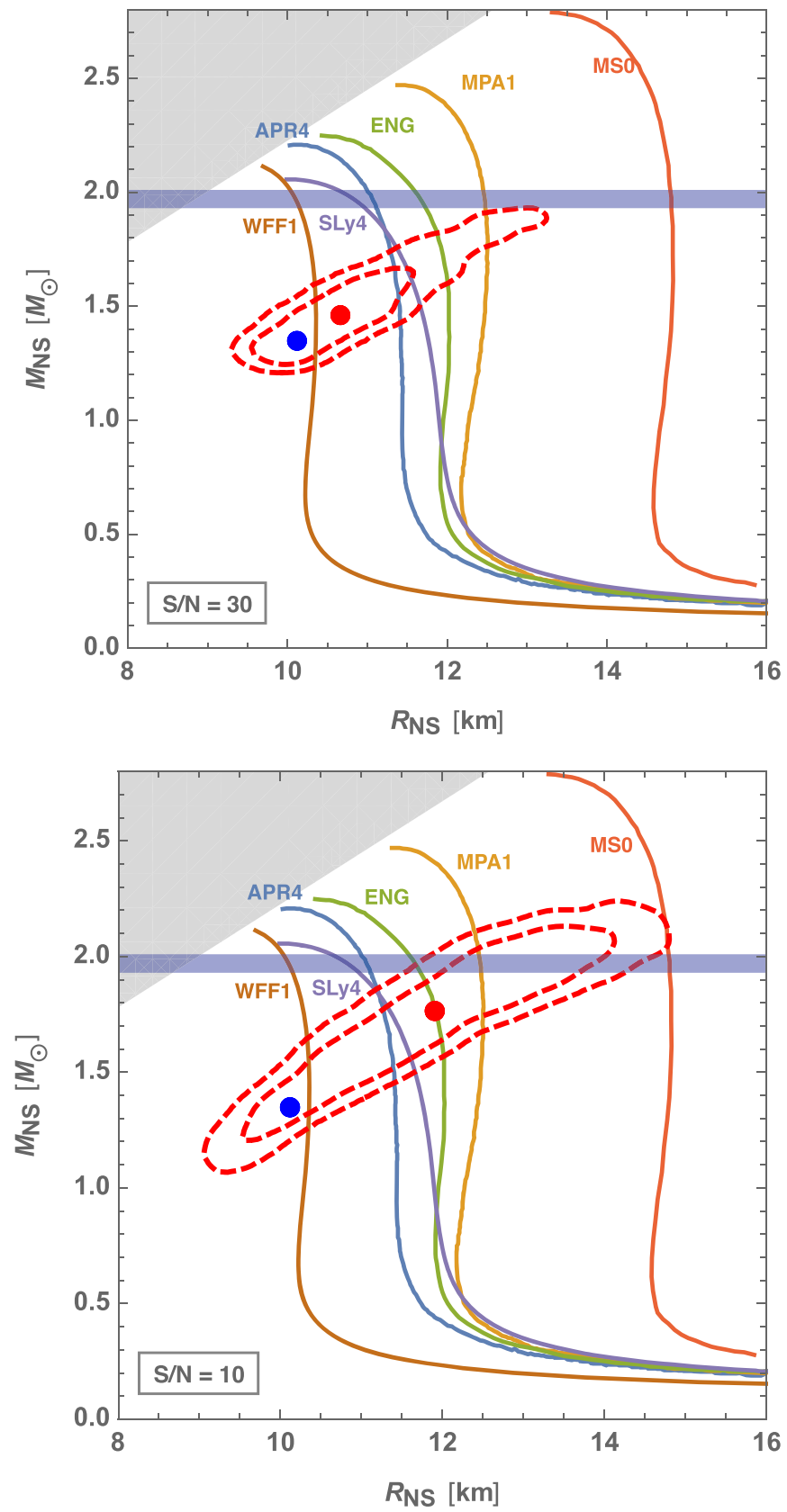

Figure 5. Same as Figure 3, but for case m484 chi $048 \mathrm{~L}$.

case. Furthermore, an $\epsilon \lesssim 0.1 \%$ gradually becomes unable to accommodate the high-energy scenario, while this is not the case for the low-energy case. Finally, the red line is the curve of constant $E_{\gamma}$ (isoenergetic curve) obtained from Equation (7) for this specific simulated scenario (i.e., for $M_{\mathrm{BH}}=4.84 M_{\odot}$, $M_{\mathrm{NS}}=1.35 M_{\odot}, \quad \chi_{\mathrm{BH}}=0.48, \quad E_{\gamma, \mathrm{ISO}}=10^{50} \mathrm{erg}, \quad \theta_{\mathrm{j}} \simeq 11^{\circ}$, $\left.c_{2}=17 / 200, \epsilon=0.01\right)$. The fact that this curve cuts through the $68 \%$ credible region shows that our analysis is capable of recovering the simulated scenario.

We now vary the injected $\mathrm{BH}$ parameters $\left(M_{\mathrm{BH}}\right.$ and $\left.\chi_{\mathrm{BH}}\right)$ to see how this affects the recovery of $R_{\mathrm{NS}}$. We begin from the $\mathrm{BH}$ spin. Figure 7 reports the results at $\mathrm{S} / \mathrm{N}=30$ for case m484chi080H. A comparison with the m484chi048H results (Figures 2 and 3, top panels) highlights that, as the $\mathrm{BH}$ spin increases from $\chi_{\mathrm{BH}}=0.48$ to $\chi_{\mathrm{BH}}=0.8$, the $R_{\mathrm{NS}}$ posterior distribution shifts to lower values, correctly 

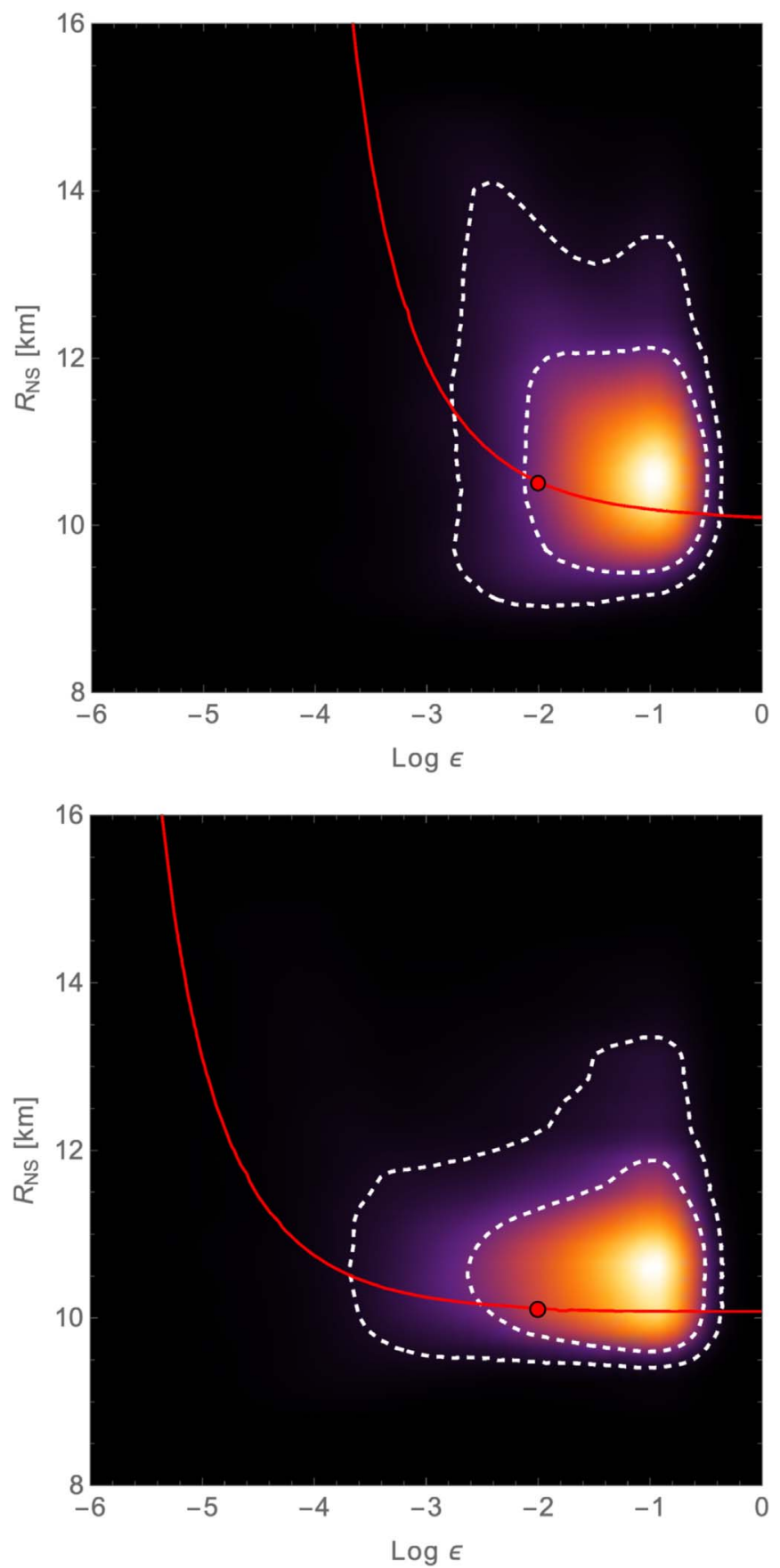

Figure 6. NS radius and mass-energy conversion efficiency $\epsilon$ constraints for case $\mathrm{m} 484 \mathrm{chi} 048 \mathrm{H}$ (top panel) and case $\mathrm{m} 484 \mathrm{chi} 048 \mathrm{~L}$ (bottom panel) with $\mathrm{S} / \mathrm{N}=30$. The white dashed curves represents the $68 \%$ and $90 \%$ credible regions, respectively. The red solid curves are the isoenergetic curve of the injection. The red dot marks the value of the injected epsilon and $R_{\mathrm{NS}}$.

following the injected $R_{\mathrm{NS}}$ value. ${ }^{17}$ In this particular case, where the value of the injected $R_{\mathrm{NS}}$ is small (see row 4 in Table 1), results are obtained by extending the prior on $R_{\mathrm{NS}}$ down to $8 \mathrm{~km}$ in order to avoid a railing of the posterior distribution against the standard boundary at $9 \mathrm{~km}$.

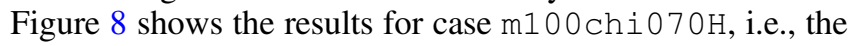
$\mathrm{BH}$ has a rather high mass and spin $\left(M_{\mathrm{BH}}=10 M_{\odot}\right.$, $\left.\chi_{\mathrm{BH}}=0.7\right)$. In this case, the $\mathrm{BH}$ mass increase requires a

\footnotetext{
17 All else being fixed, an increase in $\chi_{\mathrm{BH}}$ requires a decrease in $R_{\mathrm{NS}}$ to maintain the SGRB energy as constant.
}
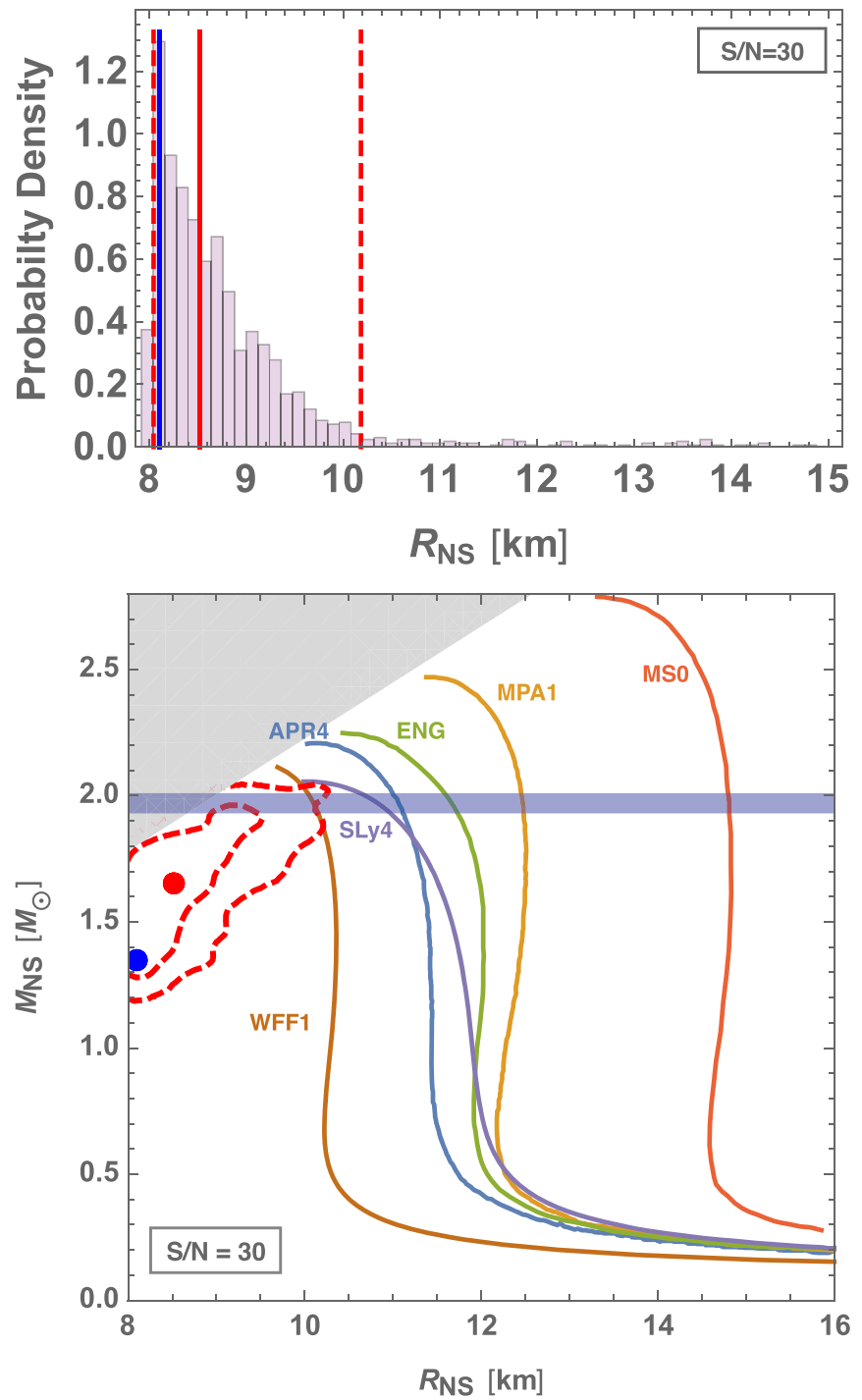

Figure 7. $R_{\mathrm{NS}}$ posterior distribution (top panel) with the $68 \%$ and $90 \%$ credible regions in the $M_{\mathrm{NS}}-R_{\mathrm{NS}}$ plane (bottom panel) for case $484 \mathrm{chi} 080 \mathrm{H}$.

higher simulated $R_{\mathrm{NS}}$ value, and the $R_{\mathrm{NS}}$ posterior distribution accordingly shifts toward higher values.

\subsection{Accuracy of the $\mathrm{R}_{N S}$ Measurement}

In this section, we address the impact of the GW posterior, which we use as an informed prior for our method, on the measurement of $R_{\mathrm{NS}}$. Furthermore, we discuss the overall uncertainty on the NS radius recovered with our approach.

Figure 9 shows cases $\mathrm{m} 484 \mathrm{chi} 048 \mathrm{H}$ and $\mathrm{m} 484 \mathrm{chi0} 48 \mathrm{~L}$ analyzed in the hypothetical scenario in which $M_{\mathrm{BH}}, M_{\mathrm{NS}}$, and $\chi_{\mathrm{BH}}$ are known exactly (which makes the $\mathrm{S} / \mathrm{N}$ value irrelevant). In other words, we set to zero any systematics deriving from the GW informed prior, but we sample $\epsilon, \theta_{\mathrm{j}}$, and $c_{2}$ normally. This allows us to quantify how the analysis of the $\mathrm{GW}$ data influences our final result. The upper and bottom panel of this figure should be compared to the panels in Figures 2 and 4, respectively. In the high-energy case, the recovered median now slightly underestimates the injected value of $R_{\mathrm{NS}}$, and the width of the posterior is reduced. The change in width of the posterior is even more dramatic for the 

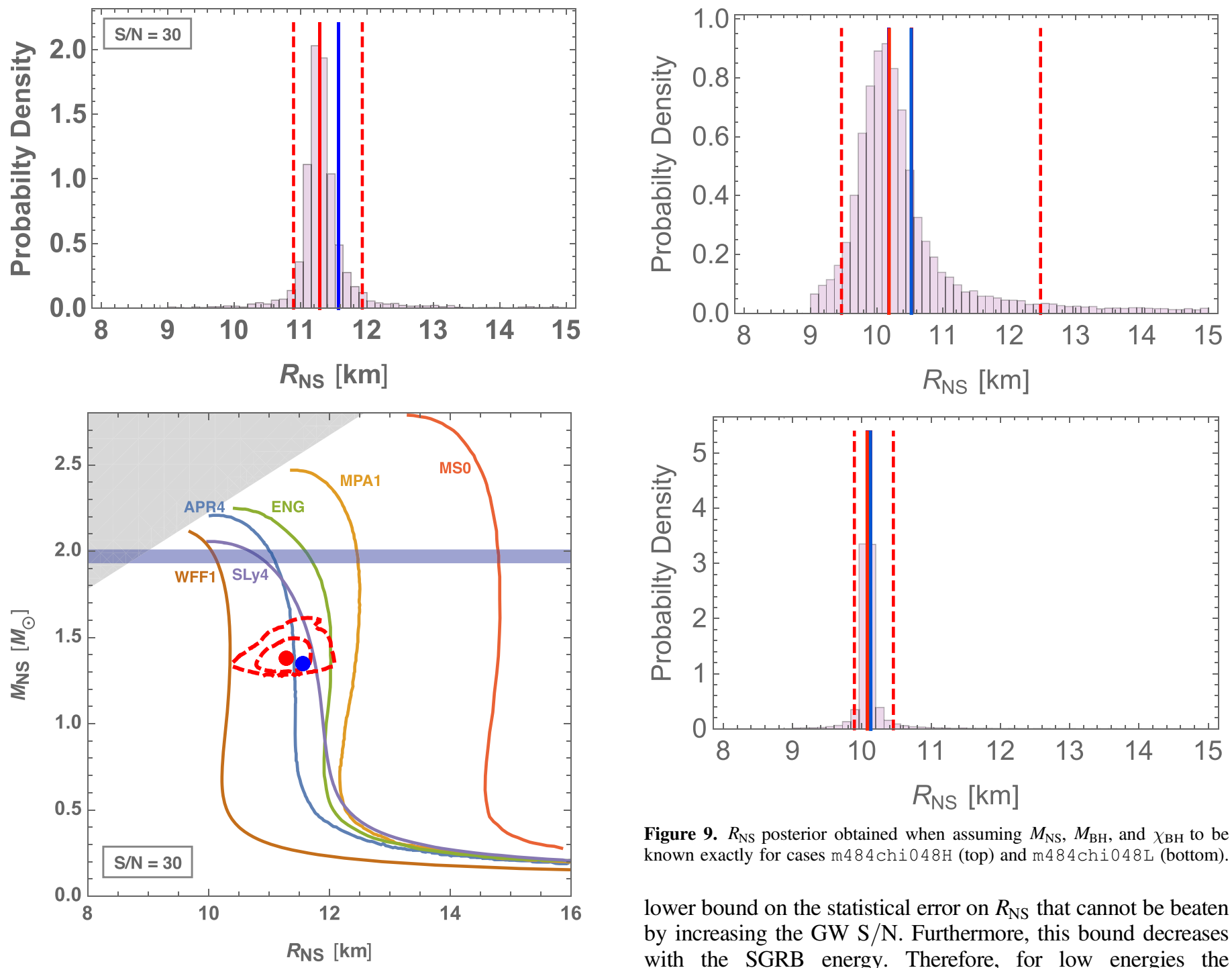

Figure 9. $R_{\mathrm{NS}}$ posterior obtained when assuming $M_{\mathrm{NS}}, M_{\mathrm{BH}}$, and $\chi_{\mathrm{BH}}$ to be known exactly for cases m484 chi048H (top) and m484 chi 048L (bottom).

lower bound on the statistical error on $R_{\mathrm{NS}}$ that cannot be beaten by increasing the GW S/N. Furthermore, this bound decreases with the SGRB energy. Therefore, for low energies the uncertainties on $M_{\mathrm{NS}}, M_{\mathrm{BH}}$, and $\chi_{\mathrm{BH}}$, which derive solely from the analysis of the GW data, end up dominating the accuracy of the measurement of $R_{\mathrm{NS}}$. The bottom panel shows that, unsurprisingly, the bias in the measurement of $R_{\mathrm{NS}}$ is larger when using the GW informed prior, as opposed to when $M_{\mathrm{NS}}$, $M_{\mathrm{BH}}$, and $\chi_{\mathrm{BH}}$ are assumed to be known exactly. As expected, the overall bias decreases with $\mathrm{S} / \mathrm{N}$. Finally, by contrasting results for which we assume to know the values of $M_{\mathrm{NS}}, M_{\mathrm{BH}}$, and $\chi_{\mathrm{BH}}$ (squares) to results that are not based on this assumption (circles), we see that the bias introduced by the GW analysis acts in the direction opposite of that of the bias introduced by the second step of our hierarchical method, i.e., sampling of $\epsilon, \theta_{\mathrm{j}}$ and $c_{2}$ and use of Equation (4).

Our lack of knowledge about $\theta_{\mathrm{j}}$ and $\epsilon$ contributes in shaping the $R_{\mathrm{NS}}$ posterior distribution. Therefore, in the event of a joint GW-SGRB NS-BH observation, any input from additional EM observations and from theoretical studies about jet-launching mechanisms could lead to improvements in the $R_{\mathrm{Ns}}$ posterior distribution. Similarly, detailed analyses of the GW alone could also improve the radius measurement further by providing a tighter informed prior for $R_{\mathrm{NS}}$ (Abbott et al. 2018a, 2019).

Finally, we wish to stress that, unfortunately, a proper assessment of all the systematics that enter our method is currently unfeasible. A first assessment of systematics could be achieved as follows. One would have to run numerical-relativity 

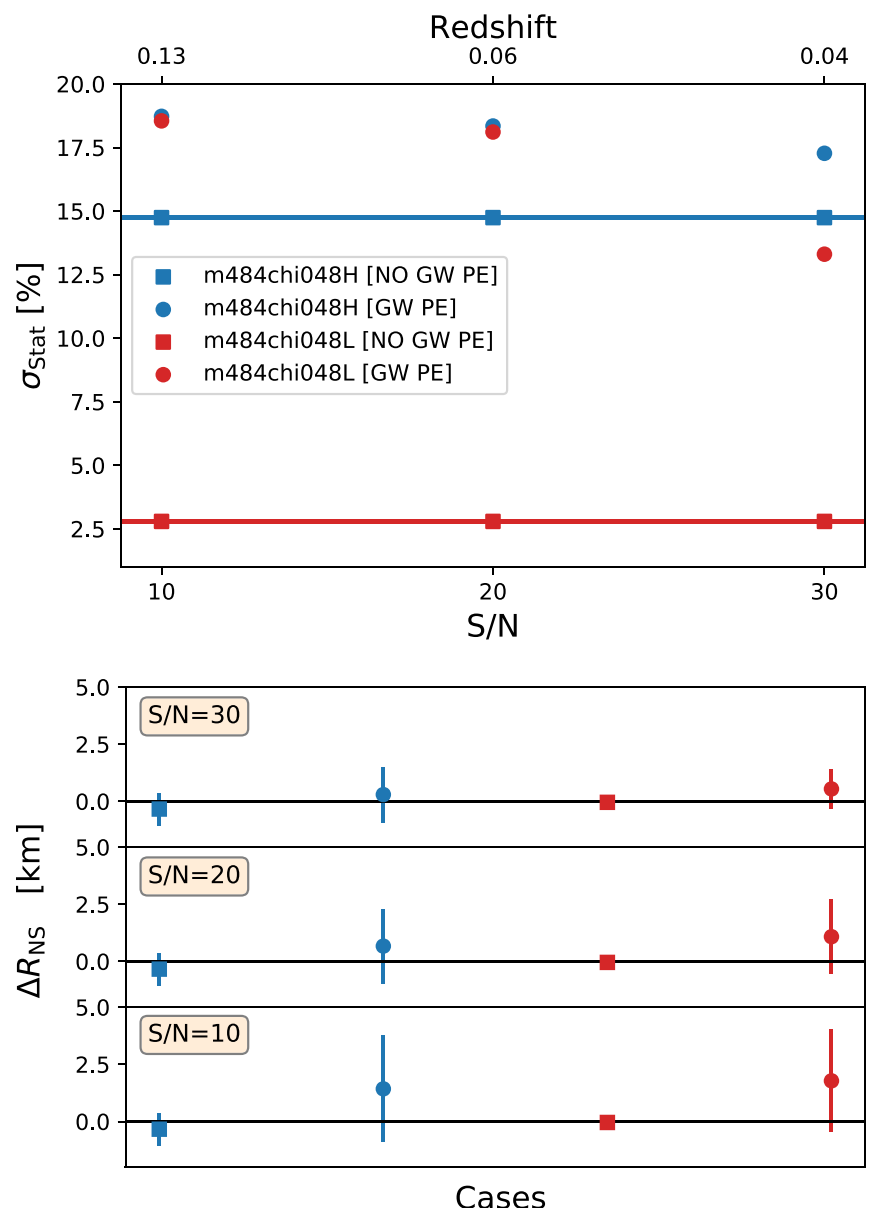

Figure 10. Top panel: statistical error (Equation (9)) on $R_{\mathrm{NS}}$ as a function of the GW S/N. The blue and red markers denote cases m $44 \mathrm{chi} 048 \mathrm{H}$ and m $484 \mathrm{chi} 048 \mathrm{~L}$, respectively. The circles (squares) represent cases that use (do not use) the prior on $M_{\mathrm{NS}}, M_{\mathrm{BH}}$, and $\chi_{\mathrm{BH}}$ informed by $\mathrm{GW}$ parameter estimation (which is denoted as "GW PE" in the legend). Bottom panel: error on $R_{\mathrm{NS}}$ for all the scenarios considered in the top panel; the symbols denote the systematic error, that is, the difference between the median and the injected value, while the bars indicate the $90 \%$ credible intervals, i.e., the statistical uncertainty.

simulations of various NS-BH mergers, extract the remnant masses from them, build complete GW signals by combining analytic approaches for the early inspiral with the numerical data for the late inspiral and merger, and finally test our method against such signals and remnant mass values. ${ }^{18}$ This extensive investigation is beyond the scope of the present work and we leave it as a topic for future studies. Because it would heavily rely on numerical-relativity simulations, this would only be a first, albeit significant step. Importantly, in this context, Foucart et al. (2018) found no systematic bias associated with the numerical-relativity code used to determine remnant mass values and that different codes predict remnant masses to within the accuracy of Equation (4).

\section{Discussion}

The joint observation of GW170817 and GRB 170817A has unambiguously associated NS-NS coalescences and SGRBs

\footnotetext{
18 All this would be done by fixing the value of $\epsilon$ in order to determine the SGRB energy, as no simulation from the initial NS-BH binary to the final SGRB is currently possible.
}

(Abbott et al. 2017b) confirming the long-standing hypothesis that NS-NS binaries are SGRB progenitors (Blinnikov et al. 1984; Paczynski 1986, 1991; Eichler et al. 1989; Narayan et al. 1992). While the rate of NS-NS mergers can accommodate for the rate of observed SGRB events (Abbott et al. 2017b), the question of whether SGRBs have more than one kind of progenitor remains an open one, and one that future observing runs of current and upcoming GW detection facilities will help answer. NS-BH systems, in particular, remain a viable SGRB progenitor candidate (see, e.g., Nakar 2007). Clark et al. (2015) determine a projected joint GW-SGRB detection rate for NS-BH coalescences of $0.1-2 \mathrm{yr}^{-1}$ for Advanced LIGO and Virgo at design sensitivity and the Fermi Gamma-Ray Burst Monitor, which decreases to $0.03-0.7 \mathrm{yr}^{-1}$ with Swift. Similarly, Regimbau et al. (2015) found a joint GW-SGRB detection rate with Swift of $0.05-0.06 \mathrm{yr}^{-1}$, while Wanderman \& Piran (2015) found $0.4-1 \mathrm{yr}^{-1}$ (3-6 $\mathrm{yr}^{-1}$ with Fermi Gamma-Ray Burst Monitor). The next generation of GW interferometers will extend the NS-BH detection horizon up to $z \simeq 4$ (Abernathy et al. 2011) therefore boosting such detection rates.

In this paper, we presented a method based on Pannarale \& Ohme (2014) to exploit joint GW-SGRB observations of NS-BH coalescences in order to measure the NS radius, and hence constrain the EOS of matter at supranuclear densities. We sample the GW posterior distribution of the component masses and the $\mathrm{BH}$ spin along with uniform prior distributions on other unknown physical parameters involved in the problem - among which is theNS radius (see Section 2 for details) and determine a distribution of isotropic gamma-ray energies. This is then combined with the EM measurement of the isotropic gamma-ray energy to yield a constraint on the NS radius, after marginalizing over all other sampled quantities. Hinderer et al. (2018) performed a similar analysis on GW170817, also using Foucart et al. (2018) and working under the assumption that the event originated from a NS-BH coalescence, but exploiting the EM constraints from the kilonova light curve, rather than the SGRB energy.

In order to test the performance and the robustness of our method, we simulated six joint GW-SGRB NS-BH detection scenarios (see Table 1). In each case, we compared the injected $R_{\mathrm{NS}}$ value to the posterior distribution recovered by our analysis. While this setup does not allow us to assess systematics in our methodology (see the discussion at the end of Section 3.5), it is currently the only possible benchmark and it allows us to draw the following first, important conclusions about our method:

1. The $90 \%$ credible regions we determine always contains the injected value of $R_{\mathrm{NS}}$, regardless of the mass and/or spin of the $\mathrm{BH}$ in the NS-BH system under consideration.

2. With the exception of case m100chi070H, the median of the $R_{\mathrm{NS}}$ posterior distribution is usually higher than the injected NS value and it is narrower for lower-energy SGRBs (i.e., $E_{\gamma, \text { ISO }} \lesssim 10^{50} \mathrm{erg}$ ).

3. We can constrain the NS radius with an uncertainty (quantified from a $90 \%$ of credible interval) below $20 \%$ even for low $\mathrm{S} / \mathrm{N}$ events.

4. The $R_{\mathrm{NS}}$ lower bound is rather solid and depends mostly on the $\mathrm{S} / \mathrm{N}$ of the $\mathrm{GW}$ signal through the informed prior for the $\mathrm{GW}$ parameters.

5. By directly sampling the posterior distributions of GW parameter estimation analyses, our method inherits any 
uncertainty that is present in such distributions. This component of the overall error on the recovered $R_{\mathrm{NS}}$ reduces as the $\mathrm{S} / \mathrm{N}$ of the $\mathrm{GW}$ increases. However, in Section 3.5 we showed that the SGRB energy determines a hard lower limit for the uncertainty on $R_{\mathrm{NS}}$. The value of this contribution to the overall error is clearly $\mathrm{S} / \mathrm{N}$ independent, but it decreases with the SGRB energy. For example, for the source configuration considered in Figure 10, this lower limit varies from

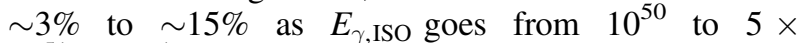
$10^{51} \mathrm{erg} \mathrm{s}^{-1}$.

A central ingredient of our method is the fitting formula that predicts the mass of the matter that remains in the surroundings of the remnant $\mathrm{BH}$ immediately after the merger as a function of the NS-BH initial parameters (Foucart et al. 2018). This can be replaced as improved or different versions of such formula are published. However, as long as it remains the only available option in the literature, a study of systematics continues to be a time and resource intensive task that would essentially require a campaign of numerical-relativity simulations (see discussion at the end of Section 3.5). Furthermore, for such a study to be fully self-consistent, one would require simulations that evolve the NS-BH system all the way from inspiral to the ignition of the SGRB. For the time being, the tolerance we introduce in Equation (6) when comparing our inferred $E_{\gamma, \text { IsO }}$ values to the observed $E_{\gamma, \text { ISO }}$ accounts for systematic uncertainties in the fit of Foucart et al. (2018), but also for possible differences between the remnant mass that it models and the disk mass that actually accretes onto the central BH. These two quantities may differ, for instance, if a non-negligible fraction of remnant mass were to be lost in form of dynamical ejecta or disk winds (Kawaguchi et al. 2016). Although our method is therefore model-dependent, we note that this is a shared feature of all other existing methods to measure NS radii (for a recent review, see Özel \& Freire 2016). For example, $R_{\mathrm{NS}}$ constraints from low-mass X-ray binary observations that are based on spectroscopic measurements of such sources in a quiescent state (Heinke et al. 2006; Webb \& Barret 2007; Guillot et al. 2011; Bogdanov et al. 2016) or after a thermonuclear burst (van Paradijs 1979; Özel et al. 2009, 2012; Güver et al. 2010a, 2010b; Güver \& Özel 2013) require, among other things, introducing assumptions about the NS atmosphere composition and magnetic field. Other methods that involve timing measurements of oscillations in accretionpowered pulsars (Poutanen \& Gierliński 2003; Leahy et al. 2008, 2009, 2011; Morsink \& Leahy 2011) require modeling the pulsed waveform and therefore depend on assumptions about NS spacetimes and other geometrical factors, such as the shape and location of the surface hotspots. Finally, EOS constraints that rely on the analysis of GW data, including our method, intrinsically depend on the waveform models used to process the GW data and on how these treat tidal effects (Abbott et al. 2018a, 2019). These examples illustrate that a model dependency is unavoidable when addressing the task of measuring NS radii. However, the availability of a number of methods each one of which relies on different assumptions and on the observation of different astrophysical systems is crucial: the combination of results that stem from various approaches can provide a more solid, final result.

On the basis of the work carried out in this paper, there are a number of lines of investigation that we plan to explore. First, in the event of an NS-BH detection, a detailed analysis of the
GW that constrains the NS tidal deformability would be carried out, as was the case for the NS-NS coalescence event GW170817 (Abbott et al. 2017f, 2018a, 2019). In turn, this information and the so-called "universal relations" (see, e.g., Yagi \& Yunes 2017 for a review) could be exploited to build a less agnostic sampling of the NS radius to be used within our approach (currently a uniform prior between 9 and $15 \mathrm{~km}$ ): upper limits on the tidal deformability would result in a narrower interval to be sampled. Moreover, this informed prior on $R_{\mathrm{NS}}$ would also ensure a more consistent sampling of the NS mass and radius, with more massive objects associated with higher compactnesses. Furthermore, in the event of an NS-BH merger observation in which the NS is disrupted by the $\mathrm{BH}$ tidal field, the GW signal is expected to shut off at a characteristic frequency which depends, among other things, on the NS EOS (Shibata et al. 2009; Kyutoku et al. 2011; Pannarale et al. 2015b). The measurement of this frequency would yield constraints on $R_{\mathrm{NS}}$ with a $10 \%-40 \%$ accuracy (Lackey et al. 2012, 2014), and we want to assess the impact of including such information into our analysis. This scenario is particularly relevant for third-generation GW detectors because the shutoff of NS-BH signals happens in the $\sim \mathrm{kHz} \mathrm{GW}$ frequency regime. The projected NS-BH detection rate for the Einstein Telescope is $\mathcal{O}\left(10^{3}-10^{7} \mathrm{yr}^{-1}\right)$ (Abernathy et al. 2011). In order to guarantee a high joint GW-SGRB detection rate of such events and to unleash the full potential they have to constrain the NS EOS, it will be of paramount importance to have functioning high-energy gamma-ray observing facilities during the lifespan of third-generation GW detectors. Finally, other independent constraints that would reduce our prior on $R_{\mathrm{NS}}$ are expected to result from ongoing and future missions such as NICER (Arzoumanian et al. 2014), ATHENA (Motch et al. 2013), and eXTP (Zhang et al. 2016).

The work presented in this article was supported by Science and Technology Facilities Council (STFC) grant No. ST/ L000962/1, European Research Council Consolidator grant No. 647839, and Cardiff University seedcorn grant AH21101018, as well as the Max Planck Society's Independent Research Group programme. We acknowledge support from the Amaldi Research Center funded by the MIUR program "Dipartimento di Eccellenza" (CUP:B81I18001170001). We are grateful for computational resources provided by Cardiff University, and funded by an STFC grant (ST/I006285/1) supporting UK Involvement in the Operation of Advanced LIGO. We thank Stephen Fairhurst and Andrew Williamson for interesting discussions throughout the genesis of this work. We also thank Michal Was for his useful comments and input. N.D.L. acknowledges support from Cardiff University's Leonid Grishchuk Summer Internship in Gravitational Physics programme. S.A. acknowledges Stefania Marassi, Silvia Piranomonte, Alessandro Papitto, Luigi Stella, Enzo Brocato, Viviana Fafone, Valeria Ferrari, and Cole Miller for useful discussions. S.A. thanks the Cardiff University School of Physics and Astronomy for the hospitality received while completing part of this work. S.A. acknowledges the GRAvitational Wave Inaf TeAmGRAWITA (P.I. E. Brocato).

\section{Appendix}

We report in Table 2 the results ( $90 \%$ of credible intervals) of the parameter estimation of the GW injected signal. The parameters considered are the masses, the spin of the primary 
Table 2

90\% Credible Intervals of Binary System Properties Obtained by the Parameter Estimation of the GW Signal Injections Used in Our Study

\begin{tabular}{lcccr}
\hline \hline Label & S/N & $\begin{array}{c}M_{\mathrm{NS}} \\
\left(M_{\odot}\right)\end{array}$ & $\begin{array}{c}M_{\mathrm{BH}} \\
\left(M_{\odot}\right)\end{array}$ & $\chi_{\mathrm{BH}}$ \\
\hline m484chi048 (H/L) & 10 & $1.25-2.46$ & $2.57-5.47$ & $0.14-0.81$ \\
m484chi080 (H/L) & 30 & $1.28-1.94$ & $3.19-5.26$ & $0.23-0.68$ \\
m100chi070 (H/L) & 30 & $1.19-2.29$ & $2.66-5.72$ & $0.65-0.88$ \\
\hline
\end{tabular}

Table 3

Same as Table 2, but Obtained from the Posterior Probability Distribution after It Is Pruned in Order to Select Only Parameters Compatible with NS-BH Systems

\begin{tabular}{|c|c|c|c|c|c|}
\hline Label & $\mathrm{S} / \mathrm{N}$ & $\begin{array}{l}M_{\mathrm{NS}} \\
\left(M_{\odot}\right)\end{array}$ & $\begin{array}{l}M_{\mathrm{BH}} \\
\left(M_{\odot}\right)\end{array}$ & $\chi_{\mathrm{BH}}$ & $q$ \\
\hline \multirow[t]{2}{*}{ m484chi048(H/L) } & 10 & $1.20-2.12$ & $2.57-5.47$ & $0.14-0.81$ & $1.45-4.83$ \\
\hline & 30 & $1.29-1.83$ & $3.05-5.84$ & $0.38-0.68$ & $1.86-4.07$ \\
\hline $\mathrm{m} 484 \mathrm{chi} 080$ (H/L) & 30 & $1.13-1.89$ & $3.30-6.17$ & $0.76-0.88$ & $1.74-5.46$ \\
\hline m100chi0 70 (H/L) & 30 & $1.32-1.53$ & $8.52-10.46$ & $0.68-0.72$ & $5.56-7.90$ \\
\hline
\end{tabular}

star and the mass ratio $q$. In Table 3 we report the same results, but obtained after the pruning of the posteriors.

\section{ORCID iDs}

Stefano Ascenzi (10) https://orcid.org/0000-0001-5116-6789 Nicola De Lillo (i) https://orcid.org/0000-0002-5083-3639 Carl-Johan Haster (i) https://orcid.org/0000-0001-8040-9807 Francesco Pannarale (iD https://orcid.org/0000-00027537-3210

\section{References}

Aasi, J., Abbott, B. P., Abbott, R., et al. 2015, CQGra, 32, 074001 Abbott, B. P., Abbott, R., Abbott, T. D., et al. 2013, arXiv: 1304.0670 Abbott, B. P., Abbott, R., Abbott, T. D., et al. 2016a, PhRvL, 116, 241103 Abbott, B. P., Abbott, R., Abbott, T. D., et al. 2016b, PhRvL, 116, 061102 Abbott, B. P., Abbott, R., Abbott, T. D., et al. 2017a, ApJL, 850, L39 Abbott, B. P., Abbott, R., Abbott, T. D., et al. 2017b, ApJL, 848, L13 Abbott, B. P., Abbott, R., Abbott, T. D., et al. 2017c, PhRvL, 118, 221101 Abbott, B. P., Abbott, R., Abbott, T. D., et al. 2017d, ApJL, 851, L35 Abbott, B. P., Abbott, R., Abbott, T. D., et al. 2017e, PhRvL, 119, 141101 Abbott, B. P., Abbott, R., Abbott, T. D., et al. 2017f, PhRvL, 119, 161101 Abbott, B. P., Abbott, R., Abbott, T. D., et al. 2017g, ApJL, 848, L12 Abbott, B. P., Abbott, R., Abbott, T. D., et al. 2018a, PhRvL, 121, 161101 Abbott, B. P., Abbott, R., Abbott, T. D., et al. 2018b, arXiv:1811.12907 Abbott, B. P., Abbott, R., Abbott, T. D., et al. 2019, PhRvX, 9, 011001 Abernathy, M., Acernese, F., Ajith, P., et al. 2011, Einstein Gravitational Wave Telescope Conceptual Design Study. ET-0106C-10, https://tds.ego-gw.it/ $\mathrm{ql} / ? \mathrm{c}=7954$

Acernese, F., Agathos, M., Agatsuma, K., et al. 2015, CQGra, 32, 024001

Akmal, A., Pandharipande, V. R., \& Ravenhall, D. G. 1998, PhRvC, 58, 1804 Arzoumanian, Z., Gendreau, K. C., Baker, C. L., et al. 2014, Proc. SPIE, 9144, 914420

Aso, Y., Michimura, Y., Somiya, K., et al. 2013, PhRvD, 88, 043007

Bardeen, J. M., Press, W. H., \& Teukolsky, S. A. 1972, ApJ, 178, 347

Berger, E. 2014, ARA\&A, 52, 43

Bildsten, L., \& Cutler, C. 1992, ApJ, 400, 175

Blandford, R. D., \& Payne, D. G. 1982, MNRAS, 199, 883

Blandford, R. D., \& Znajek, R. L. 1977, MNRAS, 179, 433

Blinnikov, S. I., Novikov, I. D., Perevodchikova, T. V., \& Polnarev, A. G. 1984, SvAL, 10, 177

Bogdanov, S., Heinke, C. O., Özel, F., \& Güver, T. 2016, ApJ, 831, 184

Chabanat, E., Bonche, P., Haensel, P., Meyer, J., \& Schaeffer, R. 1998, NuPhA, 635, 231

Cipolletta, F., Cherubini, C., Filippi, S., Rueda, J. A., \& Ruffini, R. 2015, PhRvD, 92, 023007

Clark, J., Evans, H., Fairhurst, S., et al. 2015, ApJ, 809, 53
Demorest, P. B., Pennucci, T., Ransom, S. M., Roberts, M. S. E., \& Hessels, J. W. T. 2010, Natur, 467, 1081

Dietrich, T., Khan, S., Dudi, R., et al. 2019, PhRvD, 99, 024029

Duez, M. D., Foucart, F., Kidder, L. E., Ott, C. D., \& Teukolsky, S. A. 2010, CQGra, 27, 114106

Eichler, D., Livio, M., Piran, T., \& Schramm, D. N. 1989, Natur, 340, 126

Engvik, L., Osnes, E., Hjorth-Jensen, M., Bao, G., \& Ostgaard, E. 1996, ApJ, 469, 794

Fairhurst, S. 2018, CQGra, 35, 105002

Fernández, R., \& Metzger, B. D. 2016, ARNPS, 66, 23

Foucart, F. 2012, PhRvD, 86, 124007

Foucart, F., Deaton, M. B., Duez, M. D., et al. 2013, PhRvD, 87, 084006

Foucart, F., Deaton, M. B., Duez, M. D., et al. 2014, PhRvD, 90, 024026

Foucart, F., Desai, D., Brege, W., et al. 2017, CQGra, 34, 044002

Foucart, F., Hinderer, T., Nissanke, S., et al. 2018, PhRvD, 98, 081501

Giacomazzo, B., Perna, R., Rezzolla, L., Troja, E., \& Lazzati, D. 2013, ApJL, 762, L18

Glendenning, N. K. 1992, PhRvD, 46, 1274

Guillot, S., Rutledge, R. E., \& Brown, E. F. 2011, ApJ, 732, 88

Güver, T., \& Özel, F. 2013, ApJL, 765, L1

Güver, T., Özel, F., Cabrera-Lavers, A., \& Wroblewski, P. 2010a, ApJ, 712,964

Güver, T., Wroblewski, P., Camarota, L., \& Özel, F. 2010b, ApJ, 719, 1807

Hannam, M., Schmidt, P., Bohé, A., et al. 2014, PhRvL, 113, 151101

Hawley, J. F., \& Krolik, J. H. 2006, ApJ, 641, 103

Heinke, C. O., Rybicki, G. B., Narayan, R., \& Grindlay, J. E. 2006, ApJ, 644, 1090

Hinderer, T., Nissanke, S., Foucart, F., et al. 2018, arXiv:1808.03836

Hinderer, T., Taracchini, A., Foucart, F., et al. 2016, PhRvL, 116, 181101

Husa, S., Khan, S., Hannam, M., et al. 2016, PhRvD, 93, 044006

Iyer, B., Souradeep, T., Unnikrishnan, C. S., et al. 2011, LIGO India, Tech. Rep. LIGO-M1100296, https://dcc.ligo.org/LIGO-M1100296/public

Kalogera, V., Berry, C. P. L., Colpi, M., et al. 2019, arXiv:1903.09220

Kawaguchi, K., Kyutoku, K., Nakano, H., et al. 2015, PhRvD, 92, 024014

Kawaguchi, K., Kyutoku, K., Shibata, M., \& Tanaka, M. 2016, ApJ, 825, 52

Khan, S., Husa, S., Hannam, M., et al. 2016, PhRvD, 93, 044007

Kokkotas, K. D., \& Schafer, G. 1995, MNRAS, 275, 301

Kulkarni, S. R. 2005, arXiv:astro-ph/0510256

Kumar, P., Pürrer, M., \& Pfeiffer, H. P. 2017, PhRvD, 95, 044039

Kyutoku, K., Ioka, K., Okawa, H., Shibata, M., \& Taniguchi, K. 2015, PhRvD, 92, 044028

Kyutoku, K., Okawa, H., Shibata, M., \& Taniguchi, K. 2011, PhRvD, 84, 064018

Kyutoku, K., Shibata, M., \& Taniguchi, K. 2010, PhRvD, 82, 044049

Lackey, B. D., Kyutoku, K., Shibata, M., Brady, P. R., \& Friedman, J. L. 2012, PhRvD, 85, 044061

Lackey, B. D., Kyutoku, K., Shibata, M., Brady, P. R., \& Friedman, J. L. 2014, PhRvD, 89, 043009

Lattimer, J. M., \& Prakash, M. 2016, PhR, 621, 127

Lattimer, J. M., Prakash, M., Masak, D., \& Yahil, A. 1990, ApJ, 355, 241

Leahy, D. A., Morsink, S. M., \& Cadeau, C. 2008, ApJ, 672, 1119 
Leahy, D. A., Morsink, S. M., \& Chou, Y. 2011, ApJ, 742, 17

Leahy, D. A., Morsink, S. M., Chung, Y.-Y., \& Chou, Y. 2009, ApJ, 691, 1235

Lee, W. H., \& Ramirez-Ruiz, E. 2007, NJPh, 9, 17

Li, L.-X., \& Paczyński, B. 1998, ApJL, 507, L59

LIGO Scientific Collaboration, Virgo Collaboration 2017, LALSuite, https:// git.ligo.org/lscsoft/lalsuite/tree/lalinference_o2, GitLab

Mészáros, P. 2006, RPPh, 69, 2259

Meszaros, P., \& Rees, M. J. 1992, ApJ, 397, 570

Metzger, B. D. 2017, LRR, 20, 3

Metzger, B. D., \& Berger, E. 2012, ApJ, 746, 48

Metzger, B. D., Martínez-Pinedo, G., Darbha, S., et al. 2010, MNRAS, 406, 2650

Morsink, S. M., \& Leahy, D. A. 2011, ApJ, 726, 56

Motch, C., Wilms, J., Barret, D., et al. 2013, arXiv:1306.2334

Müller, H., \& Serot, B. D. 1996, NuPhA, 606, 508

Müther, H., Prakash, M., \& Ainsworth, T. L. 1987, PhLB, 199, 469

Nakar, E. 2007, PhR, 442, 166

Narayan, R., Paczynski, B., \& Piran, T. 1992, ApJL, 395, L83

Nissanke, S., Kasliwal, M., \& Georgieva, A. 2013, ApJ, 767, 124

Oppenheimer, J. R., \& Volkoff, G. M. 1939, PhRv, 55, 374

Özel, F., \& Freire, P. 2016, ARA\&A, 54, 401

Özel, F., Gould, A., \& Güver, T. 2012, ApJ, 748, 5

Özel, F., Güver, T., \& Psaltis, D. 2009, ApJ, 693, 1775

Paczynski, B. 1986, ApJL, 308, L43

Paczynski, B. 1991, AcA, 41, 257

Pannarale, F., Berti, E., Kyutoku, K., Lackey, B. D., \& Shibata, M. 2015a, PhRvD, 92, 084050

Pannarale, F., Berti, E., Kyutoku, K., Lackey, B. D., \& Shibata, M. 2015b, PhRvD, 92, 081504

Pannarale, F., Berti, E., Kyutoku, K., \& Shibata, M. 2013, PhRvD, 88, 084011
Pannarale, F., \& Ohme, F. 2014, ApJL, 791, L7

Pannarale, F., Rezzolla, L., Ohme, F., \& Read, J. S. 2011a, PhRvD, 84, 104017

Pannarale, F., Tonita, A., \& Rezzolla, L. 2011b, ApJ, 727, 95

Parfrey, K., Giannios, D., \& Beloborodov, A. M. 2015, MNRAS, 446, L61

Poutanen, J., \& Gierliński, M. 2003, MNRAS, 343, 1301

Punturo, M., Abernathy, M., Acernese, F., et al. 2010, CQGra, 27, 194002

Regimbau, T., Siellez, K., Meacher, D., Gendre, B., \& Boër, M. 2015, ApJ, 799, 69

Sari, R., Piran, T., \& Halpern, J. P. 1999, ApJL, 519, L17

Sathyaprakash, B. S., Bailes, M., Kasliwal, M. M., et al. 2019, arXiv:1903. 09277

Setiawan, S., Ruffert, M., \& Janka, H.-T. 2004, MNRAS, 352, 753

Shibata, M., Kyutoku, K., Yamamoto, T., \& Taniguchi, K. 2009, PhRvD, 79, 044030

Smith, R., Field, S. E., Blackburn, K., et al. 2016, PhRvD, 94, 044031

Tolman, R. C. 1939, PhRv, 55, 364

Unnikrishnan, C. S. 2013, IJMPD, D22, 1341010

Vallisneri, M. 2000, PhRvL, 84, 3519

van Paradijs, J. 1979, ApJ, 234, 609

Veitch, J., Raymond, V., Farr, B., et al. 2015, PhRvD, 91, 042003

Wanderman, D., \& Piran, T. 2015, MNRAS, 448, 3026

Webb, N. A., \& Barret, D. 2007, ApJ, 671, 727

Wiringa, R. B., Fiks, V., \& Fabrocini, A. 1988, PhRvC, 38, 1010

Yagi, K., \& Yunes, N. 2017, PhR, 681, 1

Zalamea, I., \& Beloborodov, A. M. 2011, MNRAS, 410, 2302

Zhang, B., Liang, E., Page, K. L., et al. 2007, ApJ, 655, 989

Zhang, S. N., Feroci, M., Santangelo, A., et al. 2016, Proc. SPIE, 9905, 99051Q 